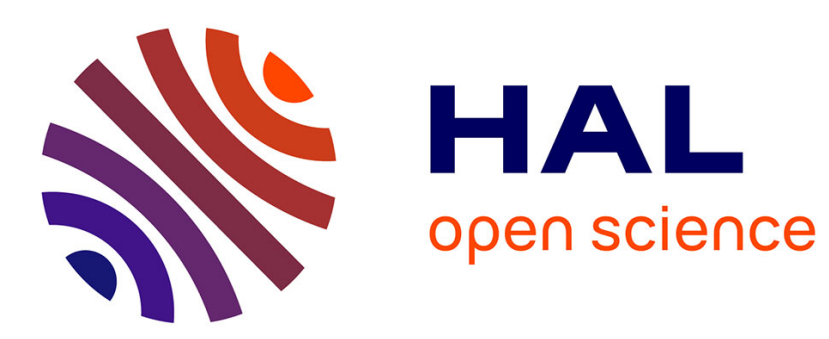

\title{
Mobile Microrobots for In Vitro Biomedical Applications: A Survey
}

Belal Ahmad, Michaël Gauthier, Guillaume Laurent, Aude Bolopion

\section{To cite this version:}

Belal Ahmad, Michaël Gauthier, Guillaume Laurent, Aude Bolopion. Mobile Microrobots for In Vitro Biomedical Applications: A Survey. IEEE Transactions on Robotics, 2021, 1, pp.1-18. hal-03433996

\section{HAL Id: hal-03433996 https://hal.science/hal-03433996}

Submitted on 18 Nov 2021

HAL is a multi-disciplinary open access archive for the deposit and dissemination of scientific research documents, whether they are published or not. The documents may come from teaching and research institutions in France or abroad, or from public or private research centers.
L'archive ouverte pluridisciplinaire HAL, est destinée au dépôt et à la diffusion de documents scientifiques de niveau recherche, publiés ou non, émanant des établissements d'enseignement et de recherche français ou étrangers, des laboratoires publics ou privés. 


\title{
Mobile Microrobots for In-vitro Biomedical Applications: A Survey
}

\author{
Belal Ahmad, Member, IEEE, Michaël Gauthier, Member, IEEE, Guillaume J. Laurent, Member, IEEE, and \\ Aude Bolopion, Member, IEEE
}

\begin{abstract}
The demand in the biomedical field for fast and precise devices for in-vitro applications has increased in recent years. Mobile microrobots are significantly suitable for such applications and are developing rapidly. These microrobots offer untethered actuation towards a contamination-free environment while allowing for fast and precise handling of biological entities for applications such as positioning, sensing, delivery, and cell surgery that are highly effective for new drug discoveries and to improve our understanding of cells behavior on the single-cell level. Here, we present a review of the recent state-of-the-art in the actuation and implementation of mobile microrobots for in-vitro applications. We will first explore the widely used methods of wireless actuation. Next, we address the challenge of implementing an on-board interaction technique to handle the target biological entity without affecting the actuation of the microrobot. Finally, we will discuss the future directions that would draw the basic outline for the next generation of mobile microrobots for in-vitro applications.
\end{abstract}

Index Terms-Micro/nano robots, automation at micronano scales, biological cell manipulation, biomedical applications.

\section{INTRODUCTION}

I $\mathrm{N}$ the past two decades, the micro world has become more accessible thanks to the drastic development of microsystems. Specifically speaking, mobile microrobots; i.e., microrobots that are powered remotely without any physical connection or wiring, with a size ranging from few microns up to a millimeter demonstrated high potential in many microscale applications due to their ability to access small and confined areas. In fact, the biomedical field is one of the major areas that mobile microrobots have shown their high potential in minimal invasive surgery [1], targeted drug delivery [2], regenerative medicine [3], and single-cell investigation [4] to

Manuscript received September 21, 2020; revised March 12, 2021; accepted May 19, 2021. This paper was recommended for publication by Editor Arianna Menciassi upon evaluation of the reviewers' comments. This work was supported by the EIPHI Graduate School (contract "ANR-17-EURE-0002"), and by the MiMedi project funded by BPI France (grant No. DOS0060162/00) and the European Union through the European Regional Development Fund of the Region Bourgogne-Franche-Comte (grant No. FC0013440). This work was also partly supported by the French ROBOTEX network and its FEMTO-ST technological facility (ANR-10-EQPX-44-01).

B. Ahmad, M. Gauthier, G. J. Laurent, and A. Bolopion are with the FEMTO-ST Institute, Univ. Bourgogne Franche-Comté, CNRS, Besançon 25000, France (e-mail: michael.gauthier@femtost.fr, guillaume.laurent@femto-st.fr, aude.bolopion@femto-st.fr). Corresponding author: B. Ahmad (email: belal.ahmad@femto-st.fr). name a few. Biomedical applications are mainly divided into two categories, applications that are conducted inside the human body, called in-vivo applications, and applications that are conducted in the laboratories outside the human body, called in-vitro applications [5]. Although mobile microrobots developed for in-vitro and in-vivo applications share a number of characteristic in their implementation such as size, remote actuation, and biocompatibility [6], [7], the difference in the targeted application and actuation environment reflects a difference in their required specifications that should be carefully considered in the design stage. For in-vivo applications, obtaining the position information of the microrobot to establish a control loop is challenging, since the microrobot will be actuated inside the human body and conventional visual feedback becomes unfeasible, contrary to in-vitro applications [8], [9]. Therefore, other techniques of position feedback should be applied by using medical imaging devices [10], [11]. In addition, the microrobot should be retrievable or biodegradable so it does not stay in the human body after the required task is accomplished [12], in contrast with in-vitro applications in which the microrobot can either be disposed or retrieved easily from the environment. On the other hand, many in-vitro applications are related to singlecells where the target size can range from few hundred micrometers such as oocytes down to few micrometers such as erythrocytes, compared to in-vivo applications that mostly deal with a group of cells such as tissues and organ samples that tend to be much larger. This difference in the targeted biological sample requires invitro microrobots to have a superior precision, compared to in-vivo counterparts, sometimes in sub-micrometer depending on the targeted cell type, which requires the establishment of highly precise actuation techniques together with control algorithms that provide accuracy and robustness. Moreover, unlike in-vivo applications where the task is conducted for one or few number of times such as extracting a sample from a tissue, in-vitro applications are more challenging in terms of throughput. Applications such as selective cell sorting and sensing, where its common to deal with millions of cells, require a high throughput through high speed actuation and feedback without sacrificing the precision. Current review papers on mobile microrobots in the biomedical field are mostly focused on in-vivo applications and microsurgery [13], [14], [15], [16], [17], [18] 
or do not target a specific application [19], [20], [21]. Therefore, this review will focus on mobile microrobots for in-vitro applications because of the need to have a critical view on the state of the art in this domain.

In recent years, the importance of in-vitro biomedical applications such as biological entities positioning, separation, sensing, stimulation, and cultivation is growing rapidly to elucidate the functions and behaviors of these entities such as its stimulus-response, function of receptors, and mechanical properties [22], [23], [24]. This kind of new knowledge can be of a great benefit in a number of highly demanding biomedical domains including regenerative medicine and drug discovery. In single-cell applications, which are one of the major areas concerning in-vitro applications, conventional methods commonly applied by biologists relied on the use of relatively large, tethered micromanipulators equipped with end effectors, such as glass micropipettes or microneedles, to perform tasks including isolation, positioning, enucleation, and injection on target cells [25]. However, the use of tethered micromanipulators necessitates highly skilled human operators working on an openspace environment such as a Petri dish, hence reducing the throughput and increasing the risk of contamination. On the other hand, cytometers have been commonly used devices to achieve cells analysis and sorting utilizing fluidic streams inside a microchannel [26], or dielectrophoresis (DEP) [27]. Although cytometers and DEPbased sorters offer a very high throughput with good accuracy, they largely depend on the cell characteristics, such as size, to achieve the sorting, which limits the ability of such devices to selectively sort cells that have similar characteristics. Therefore, the development of new tools for in-vitro applications that allow a better handling and characterization of single-cells is essential.

The use of mobile microrobots for in-vitro applications offers a large number of benefits compared to previous methods. In fact, the small size and relatively simple manipulation of mobile microrobots provides high repeatability and throughput. Additionally, thanks to the versatility, adaptability, and remote actuation of mobile microrobots, they are able to interact with individual biological entities, such as cells, that fall into their size range of handling regardless of their characteristics. Applications that demand versatility such as the selective sorting of cells with different characteristics can be applied inside a low contamination closed environment. The huge advancement in the fabrication techniques of microelectromechanical systems (MEMS) and nanofabrication allows for exploiting the advantages of microrobots in the biomedical field more than ever before. Techniques such as standard photolithography and multiphoton lithography are widely used to produce precise micro and nano structures with functional elements that can function as microrobots [28], [29]. All of the mentioned advantages make mobile microrobots a strong candidate technology for in-vitro applications including but not limited to single-cell positioning, cell

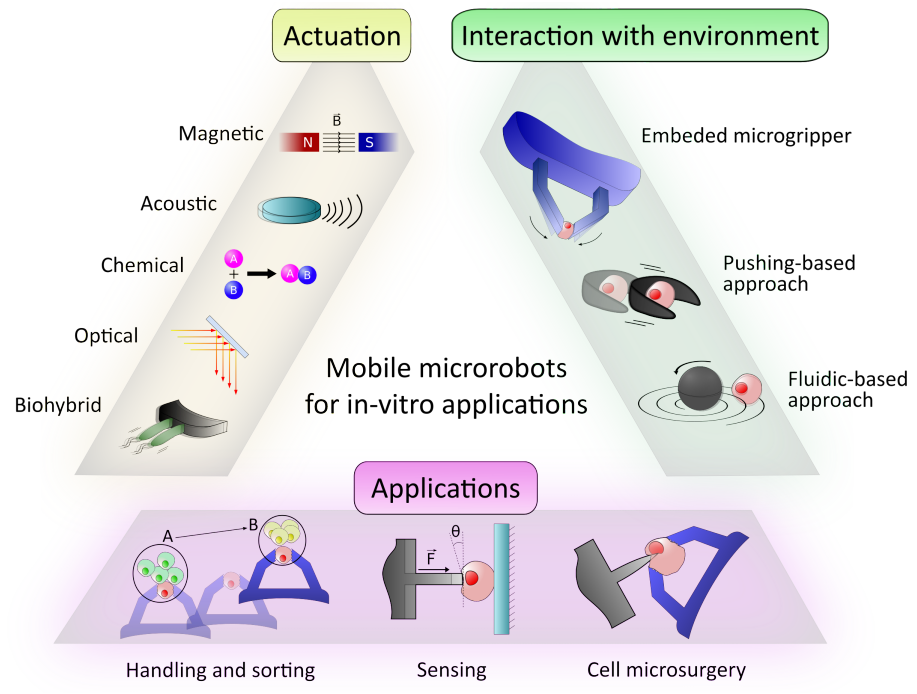

Fig. 1. Conceptual outline depicting the different actuation techniques, environment interaction approaches, and applications of mobile microrobots for in-vitro biomedical applications.

sorting, cell surgery, and pathogens sensing.

This review, will provide a comprehensive and technical survey for the use of mobile microrobots for invitro applications. Based on our knowledge, this review paper is the first one focusing on the description of the scientific paradigm and the results of microrobots for in-vitro applications. Figure 1 demonstrates a graphical summary emphasizing the main areas covered by this review, which will be organized as follows: In section 2, the different techniques for remotely actuating the microrobots, mainly magnetic, acoustic, optical, chemical, and biohybrid actuation approaches will be briefly introduced and compared. In section 3 , the implementation of the interaction approaches between the microrobot and its environment will be discussed. In section 4, a number of high-impact in-vitro applications carried out by mobile microrobots will be introduced. Finally, in section 5 , summary and future directions for the development of mobile microrobots for in-vitro applications will be shown.

\section{Actuation}

Unlike conventional tethered microrobots, mobile microrobots need to be actuated remotely with no physical connections to the microrobot. There exist a number of actuation techniques to transfer a driving energy to the microrobot in a tetherless fashion, each having its own advantages and disadvantages. In this section, we will review the main five actuation techniques for mobile microrobots covering magnetic, acoustic, chemical, optical, and biohybrid actuation by explaining the principle behind each technique with comprehensive examples from recent literature regardless of the targeted application. 


\section{A. Magnetic Actuation}

Magnetic actuation is the most widely used technique to actuate mobile microrobots owing to its versatility and ease of implementation. Moreover, magnetic fields up to a defined strength are biocompatible with no harmful effect on cells and biological tissues, which makes it an appropriate choice for biomedical applications [30]. Microrobots actuated by magnetic fields can be translated and rotated with a variety of degrees of freedom (DOF) depending on the magnetic system setup. A microrobot consisting of a magnetic material in an external nonuniform magnetic field experiences both a torque $\vec{\tau}_{m}$ and a force $\vec{F}_{m}$ :

$$
\begin{gathered}
\vec{\tau}_{m}=V(\vec{M} \times \vec{B}) \\
\vec{F}_{m}=V(\vec{M} \cdot \nabla) \vec{B}
\end{gathered}
$$

where $V$ is the volume of the microrobot, $\vec{M}$ is its magnetization vector, and $\vec{B}$ is the magnetic field. The torque is a result of the tendency to align the magnetization axis of the microrobot with the applied magnetic field direction. This torque can be used for orientation control of microrobots, or to produce non-reciprocal motion to propel microswimmers by using a time-varying magnetic field. On the other hand, the force is a result of the magnetic field gradient, where a microrobot tends to be attracted to the regions with a higher magnetic field strength, which generates a translational motion. The manipulation of a submillimeter microrobot by using a combination of magnetic torques and forces is widely applied. Magnetic actuation can be categorized based on the source of the magnetic field to actuation using electromagnets and actuation using permanent magnets. Both of these techniques have been used for planar twodimensional (2D) and three-dimensional (3D) actuation of microrobots with up to 6DOF; i.e. translation and rotation over all three Cartesian axis, [31], [32], [33].

Electromagnets are well-suited for applications that require a high degree of control over the magnetic force and torque components. Using a set of electromagnets, the magnetic field strength and direction can be controlled by controlling the amplitude and frequency of the currents supplying the coils. This high degree of controlability makes electromagnets suitable for implementing both $2 \mathrm{D}$ and $3 \mathrm{D}$ actuation. In $2 \mathrm{D}$ actuation, the magnetic gradients are used directly to translate the microrobot. In this case, the surface area of the interface between the microrobot and the substrate plays an important role in defining the adhesion forces acting on the microrobot that are caused by the dominance of van der Waals forces and surface forces in the microscale [34], [35], [8], [36] (Fig. 2a). Therefore, the use of rotating magnetic fields to generate a rolling motion or magnetic torques to generate a stick-slip motion can be implemented to overcome adhesions [37]. Additionally, modular microrobots were developed for different locomotion modes such as snake-like motion [38], pivot walking, tumbling, and tapping [39], which showed highly promising solutions in overcoming surface forces. As for 3D actuation, helical and bioinspired microrobots have been actuated using rotating magnetic fields generated by electromagnets. In helical microrobots, the rotating fields induce a corkscrew-like motion to generate a propulsive force [40], [41] (Fig. 2b). Moreover, the independent control of two helical microrobots was also reported by utilizing transchiral microrobots [42]. By using two helices with opposing handedness connected by a rod, a frequency dependent velocity profile can be realized and used to drive each microrobot independently [43]. On the other hand, bioinspired microrobots take the advantage of the efficient bacterialike motion in fluids with low Reynolds number [44], [45], [46]. These microrobots include microorganism-like cilia or bacteria-like flagella fabricated with different magnetization directions to generate a preprogrammed motion using magnetic fields [47], [48]. Maier et al. [49] have fabricated a flagella-based microswimmer using DNA-based self-assembly combined with biocompatible magnetic microparicles (Fig. 2c). Using a rotating magnetic field, the bacteria-like motion was achieved by means of flagellar bundles. Moreover, self-assembled nanoparticles have been developed to form a bacterialike structure exhibiting a flexible non-reciprocal motion under rotating magnetic fields [50].

Although the use of electromagnets is a well established and is an attractive approach, the need to use high driving electrical currents to generate a relatively large pushing force in the order of millinewton is not power efficient and results in overheating. Therefore, for applications requiring large pushing forces, permanent magnets have been utilized to drive microrobots in $2 \mathrm{D}$ with 3DOF, and rotating permanent magnets in 3D with 5DOF actuation [51], [52]. Hagiwara et al. [51] have presented a high-force and high-speed mobile microtool driven by horizontally arranged permanent magnets placed under the microtool. Using this approach, they succeeded in 3DOF actuation with a millinewtonorder generated force, and the microtool was applied for oocyte positioning, which is considered one of the large cells that requires a large pushing force.

One of the important aspects when using magnetically actuated microrobots for biomedical applications is the biocompatibility of the magnetic material composing the microrobot. In fact, there exist a number of biocompatible magnetic materials, although the biocompatibility degrades with time. Hence, the microrobot can be coated with a biocompatible layer such as polymers [53], which can also contribute to the reduction of the effect of adhesion in case of $2 \mathrm{D}$ actuation or when the microrobot is in contact with target cells or objects. Moreover, biocompatible hybrid cells were fabricated by incorporating superparamagnetic particles inside human breast cells using centrifugal force [54]. The position of the artificial cells was controlled using magnetic feedback control showing a promising solution for biocompatible magnetic microrobots. Overall, despite some of its limi- 

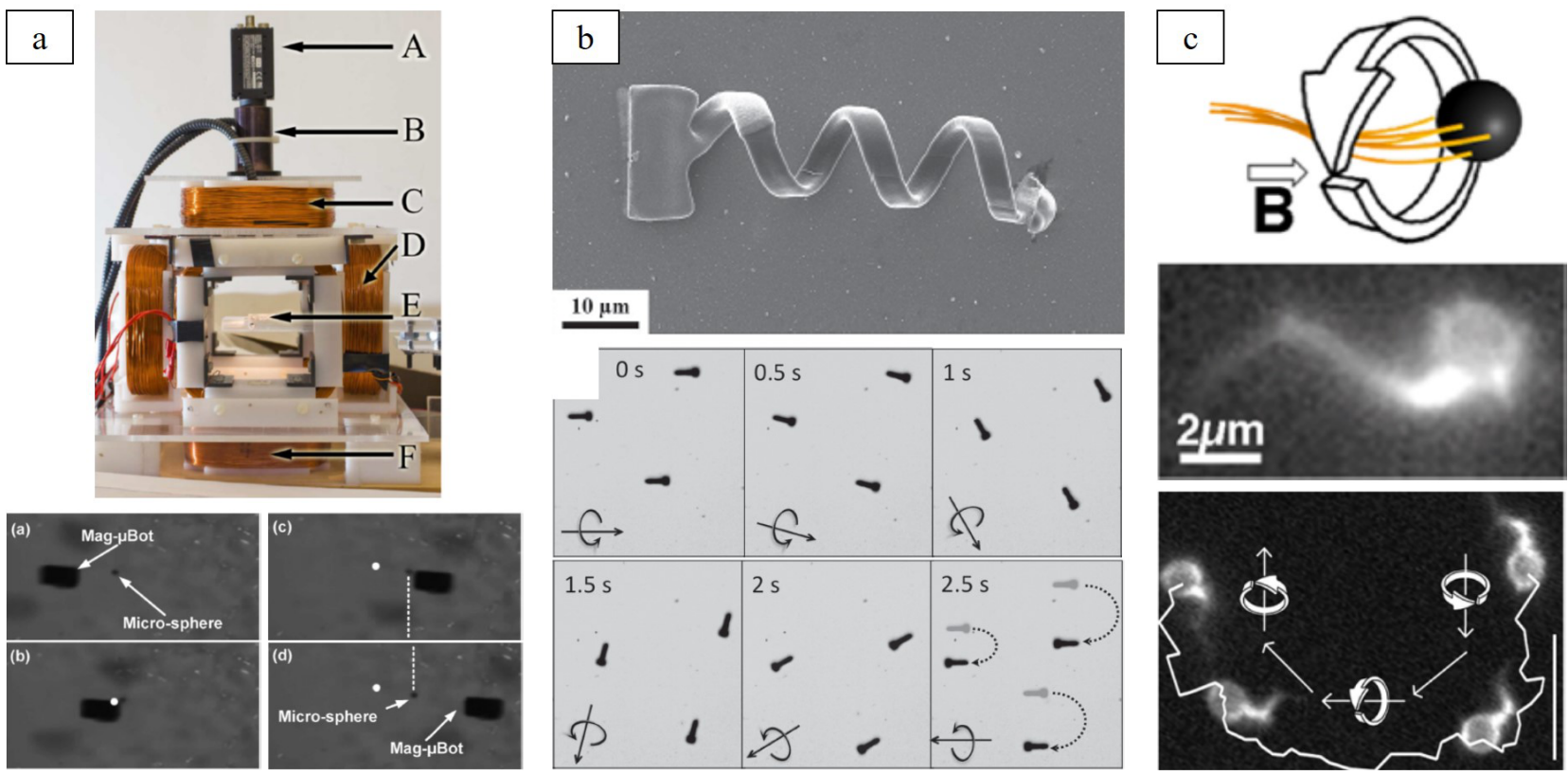

Fig. 2. Magnetic actuation. (a) 2D manipulation of a mobile microrobot utilizing stick-slip motion (reprinted with permission from [37]). (b) A magnetic helical microrobot and the actuation of a swarm-like agents in different directions by turning the rotational axis of the magnetic field (reprinted with permission from [40]). (c) DNA artificial flagella attached to a magnetic microparticle and rotated using rotating magnetic fields to induce controlled motion (reprinted with permission from [49]).

tations, magnetic actuation of mobile microrobots is still one of the promising and widely used techniques to this day.

\section{B. Acoustic Actuation}

Acoustic actuation is another attractive and biocompatible method to remotely actuate and functionalize microrobots. In this case, instead of magnetic or electromagnetic fields, acoustic vibration fields are utilized to generate vibrations and hence movements in mobile microrobots. In fact, acoustic fields are widely implemented in two ways, traveling waves and standing waves, where traveling waves are more dominantly used to allow microbubbles or artificially fabricated flagella integrated in the body of the microrobot to vibrate and produce motion.

Microrobots containing microbubbles are usually actuated by identifying the resonance frequency of the microbubble, where large oscillations could be utilized to generate movements [57]. For instance, Ren et al. proposed a microbubble-based microrobot capable of controllable high-speed swimming and moving through 3D obstacles [55] (Fig. 3a). The acoustic waves generated by a transducer caused vibrations in the microbubble, hence inducing fluidic streams for propulsion. The tilt angle of the microrobot could be changed to control the swimming speed by virtue of a nickel layer that interacts with an external permanent magnet, where a remarkable speed of 350 body lengths per second was reached. In addition, a surface-slipping microrobot that can move on both flat and curved surfaces unidirectionally with high speed and controllability was developed by integrating a fin on the surface of the microrobot [58]. Moreover, delivery and manipulation of drugs using acoustically activated microbubbles have been demonstrated for a magnetically actuated microrobot with functionalized microbubbles [59]. The acoustically activated microbubbles integrated inside the body of the microrobot could be used to release the drug and to generate a fluidic flow that would increase the absorption of the drug by the targeted tissue. Although this work was targeted to in-vivo applications, the same principle could be used to target cells in-vitro.

Recently, bioinspired acoustically driven microrobots are being explored to exploit the advantages of the motion of swimming microorganisms in fluids with low Reynolds number [60], [56]. For instance, Kayank et al. [56] developed acoustically actuated microrobots equipped with flagella-like artificial tails that demonstrate translational or rotational motion depending of the shape of the microrobot (Fig. 3b). The acoustic transducer generates traveling waves that induce oscillations in the artificial tails and results in the swimming motion of the microrobot. One of the major drawbacks of acoustic actuation is its low selectivity, since the acoustic waves would affect all the objects in the working area. To tackle this limitation, Fei et al. [61] proposed a single beam acoustic radiation force using a needle type ultrasonic transducer. The focused acoustic beam was able to individually, and stably manipulate $15 \mu \mathrm{m}$ microbeads without affecting nearby beads. However, the ultrasonic transducer had to be placed inside the fluidic environment, which restricts its use to open-space applications. Acoustic actuation is an emerging tech- 


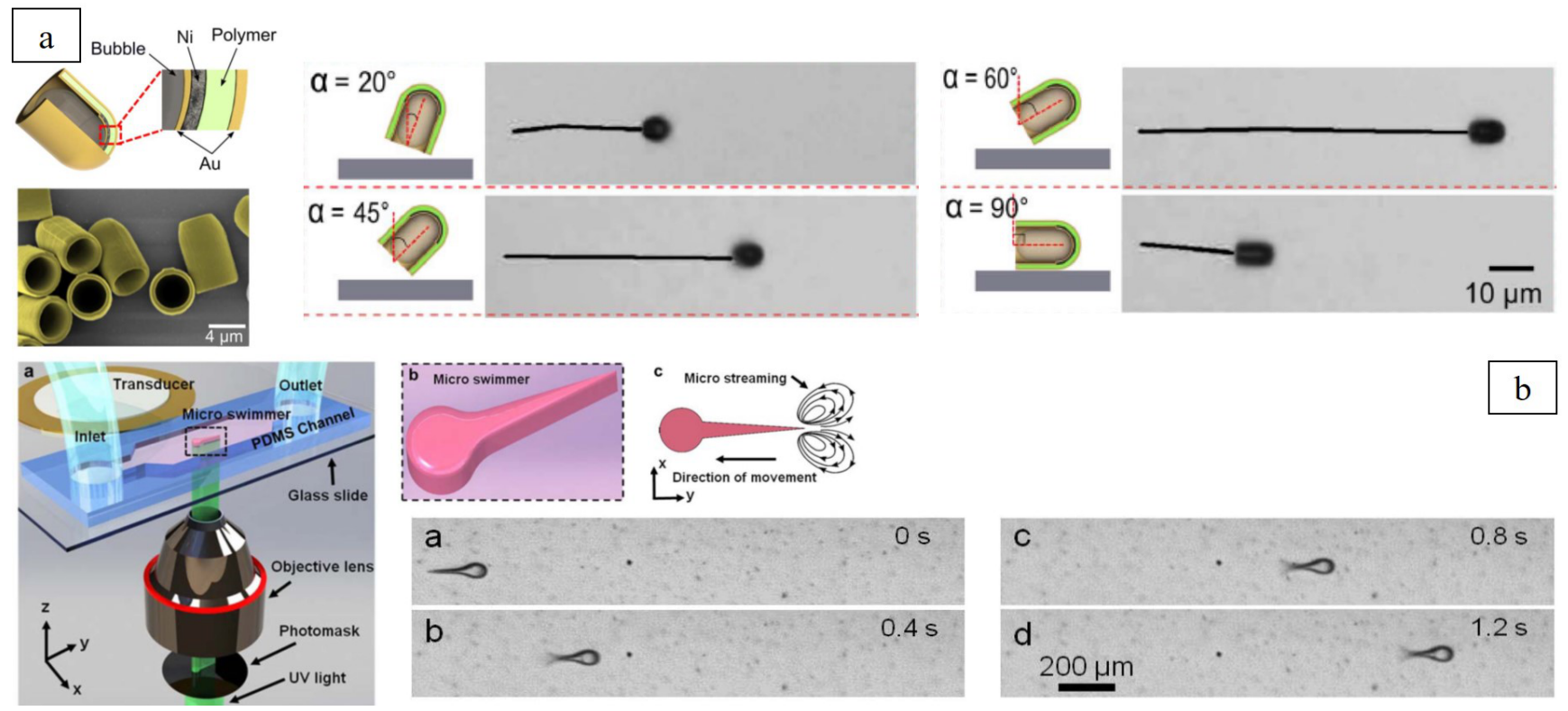

Fig. 3. Acoustic actuation. (a) Bubble-based acoustic microrobot with controllable tilt angle and moving speed (reprinted with permission from [55]). (b) Actuation of a microrobot equipped with flagella-like artificial tails to generate fluidic streams and a consequent motion upon acoustic excitation (reprinted with permission from [56]).

nique for the actuation of mobile microrobots, although its application in the biomedical experiments is still limited, which leaves a space for future developments and potential breakthroughs.

\section{Chemical Actuation}

Chemical-based actuation is an attractive technique to drive mobile microrobots owing to its on-board nature, i.e. the microrobot produces its own propulsive force, and the high selectivity of chemical reactions. The basic principle is that the microrobot uses its own environment as a fuel source to induce a chemical reaction that would generate a propulsive energy strong enough to translate the microrobot. Unlike the macroscale, where an object can counter the viscous drag and maintain its motion for a certain period after the propulsion is terminated thanks to its inertia, the continuous generation of propulsive force is crucial for chemically actuated microrobots in the microscale to overcome the dominant viscous drag force. The most widely used approach to implement chemically propelled microrobots is to integrate a catalytic material in the composition of the microrobot that interacts with the surrounding fluidic environment such as hydrogen peroxide to generate bubbles. The generation of bubbles and the resulting propulsive force causes a directional translation of the microrobot. The size of microrobots propelled by the generation of bubbles ranges between few to tens of micrometers.

In fact, microtubes and Janus micromotors are promising and widely used approaches for self-propelled microrobots that are capable of the continuous generation of bubbles. In the case of microtubes, the inner surface of the tube is composed of a chemically active material that would interact with the fluidic environment and generate bubbles internally that are ejected from the nozzle to propel the microtube [62], [63], [64], [65]. Hu et al. have implemented the rolled up nanomembrane technology to fabricate state-of-the-art layer-by-layerassembled microtubes that are chemically propelled [66] (Fig. $4 \mathrm{a}(\mathrm{I})$ ). By utilizing platinum nanoparticles as an active material, they achieved rapid speed propulsion for cell transport at high speed. This type of self-proppelled microswimmers can reach another level of functionality by utilizing $3 \mathrm{D}$ printing technology to fabricate more complex shapes. Ceylan et al. proposed a novel bulletshaped 3D microswimmer that incorporates a functional cavity with a nozzle acting as an engine [67]. A two-step fabrication process utilizing the spatiotemporal control of two-photon crosslinking was used to selectively pattern the cavity compartment with platinum nanoparticles allowing for bubble generation through the nozzle for propulsion. Janus micromotors implement a sphere with usually an outer layer of titanium dioxide covering half of the sphere and an inner layer of a reducing agent. The opposite approach that implements the titanium dioxide as the inner layer of a hemisphere has also been reported [68], [69]. Similar to the microtube approach, when the sphere is inserted in an aqueous peroxide, bubbles are generated and the propulsion is achieved.

In order to control the trajectory and speed of chemical-based microrobots, a complementary actuation have to be used since chemical actuation is only used for generating propulsive energy. For example, orientation and steering control can be achieved using external magnetic fields by integrating a ferromagnetic material in the composition of the microrobot [70], [71], [72]. In addition, dynamic speed control of microrobots using 
external force fields has also been demonstrated. For instance, the speed control as well as on/off control of a microtube with fast changes in the speed within less than $0.1 \mathrm{~s}$ were achieved by disrupting the generation and ejection of bubbles inside a microtube using ultrasound transducers [73] (Fig. 4a(II)). Moreover, a number of Janus particles can be functionalized to self-assemble under the influence of a magnetic field in one functional microrobot to generate an accumulated force able to handle cells that are an order of magnitude larger than individual particles [74]. On the other hand, the majority of chemically propelled microrobots utilize toxic fuels that may affect the viability of cells and further work is required to exploit biocompatible fuels [75]. In summary, although chemical actuation is mainly limited to generating propulsion for mobile microrobots and offers low biocompatibility, it is well suited for applications that demand on-board and high-speed propulsion.

\section{Optical Actuation}

Optical actuation in the micro world has developed drastically in the past four decades since the invention of optical tweezers. In the biomedical field, using light to actuate mobile microrobots shows a significant potential because of the transparent nature of the environment in in-vitro applications. Compared to other remote actuation techniques, optical actuation is highly selective and precise, which allows for simple and uncoupled actuation of microrobotic agents or targeted excitations of specific parts of a microrobot. In fact, optical actuation can be used as a trapping technique [78], as an optothermal source [76], [79], [80], or as a stimuli for soft active materials [81]. An optical trap can be generated by tightly focusing a laser beam using an objective lens. Consequently, a trapping force can be exerted on a dielectric particle at the beam waist caused by the momentum from the scattering of incident photons. $\mathrm{Wu}$ et al. [78] developed an optical system to actuate and spin a birefringent microsphere for directional control of the growth of axons. Both translational and rotational actuations were achieved using optical traps and a circularly polarized light with angular momentum, where the shear force resulting from the rotational motion caused the axon to turn in the desired direction. Moreover, because conventional optical trapping methods are susceptible to scattering and are dependent on the direction and the shape of the beam, asymmetrical particles that are controlled by changing the frequency of the incident light were introduced [82]. In this case, an optically induced thermophoretic drift propels a towfaced nanoparticle with two different materials. Because the two materials have different resonant absorption spectra, a local thermal gradient can be generated and controlled by the light frequency even in highly scattering environments. However, optical trapping is limited to piconewton force generation and the manipulation of small size objects up to few micrometers, hence limiting its applications for cell positioning.
On the other hand, Optically induced thermal gradients at the air-liquid interface would generate a directional fluidic flow to the cooler regions. This principle can be utilized to create and actuate microbubbles, which acts as the air-liquid interface (Fig. 4b). The position of one or multiple microbubbles can be controlled to push and manipulate microobjects by changing the position of the focused laser beam and the consequent thermal gradient [76]. However, this approach has been only demonstrated in 2D and requires the implementation of a special substrate in the environment that generates heat by optical stimulation.

Moving away from spherical objects, the optothermal stimulus-response of soft active materials such as liquid crystal elastomers has been utilized to fabricate non-spherical optically actuated microrobots [81]. Biomimetic translational or rotational motion through traveling-waves can be realized by exposing the microrobot to a structured monochromatic light. Overall, the high accuracy and selectivity of optical actuation makes it one of the successful actuation methods available for mobile microrobots taking in mind the limitations in size and generated force.

\section{E. Biohybrid Actuation}

Swimming microorganisms and bacteria have been around since billions of years and have evolved to be experts in swimming at the microscale. In fact, microorganisms can swim at relatively high speeds and possess a number of taxis that acts as sensors to navigate the surrounding environment. Therefore, combining microorganisms with microrobots proved to be a very attractive approach to navigate in microfluids. The basic principle here is to attach a microorganism to the body of the microrobot to act as an on-board actuator or a sensor. To achieve that, a number of techniques that utilize the electrostatic interaction [83], [84], chemical interaction [85], or physical entrapment have been developed to attach microorganisms to microrobots [77], [17] (Fig. $4 \mathrm{c})$. Moreover, controlled adhesion of microorganisms to target one part of the microrobot to enhance the controllability of biohybrid microrobots has also been demonstrated [86], [87]. Despite the great advantages offered by the biohybrid approach, the role of the attached bacteria is mostly restricted to propulsion, where the steering of the microrobot is mainly achieved through exploiting the different taxis of the microorganisms or using other steering techniques such as magnetic fields [85], [88], [77], [89].

Table I summarizes the pros and cons of each of the actuation technique demonstrated in this section. A variety of actuation techniques have been developed for the actuation of mobile microrobots. Each of the techniques is suitable for a set of applications depending on the required specifications and should be considered at the design stage. These techniques are also used to facilitate the interaction between the microrobot and 


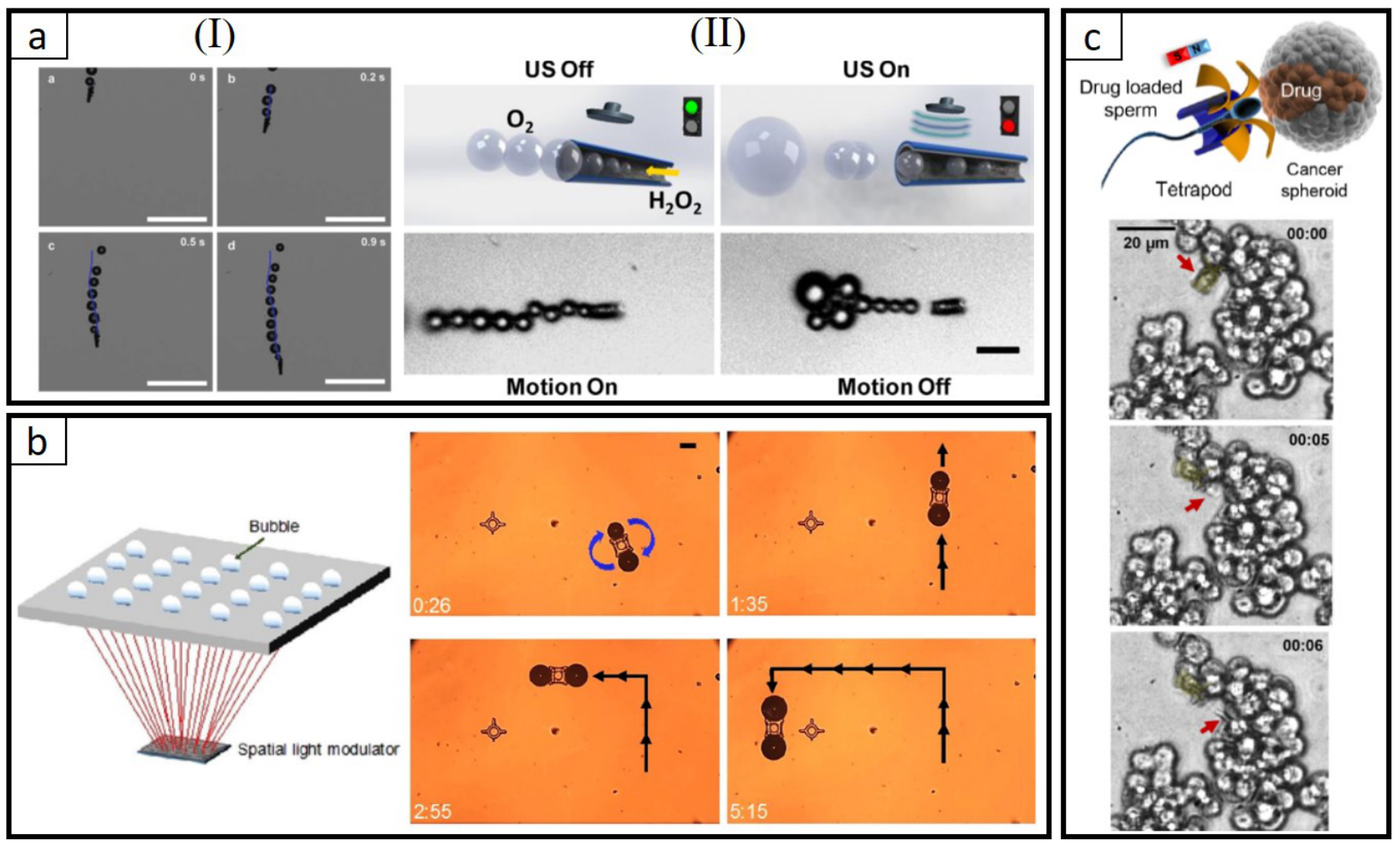

Fig. 4. Chemical, optical, and biohybrid actuation. (a) Chemical actuation (I) High speed chemical propulsion of a microtube utilizing platinum nanoparticles for bubbles generation (reprinted with permission from [66]). (II) Ultrasound modulation of bubbles generation for speed control of a chemically propelled microtube (reprinted with permission from [73]). (b) Actuation of multiple microbubbles by optically induced thermal gradients (reprinted with permission from [76]). (c) Biohybrid microrobot for targeted drug delivery using drug-loaded trapped sperm cells and guided by a magnetic field (reprinted with permission from [77]).

the target cells, where the choice of one technique over the other will be based on factors such as complexity, required DOF, and required force as will be discussed in the following section.

\section{INTERACTION WITH THE ENVIRONMENT}

In this section, the different approaches implemented in mobile microrobots to interact with their environment will be discussed. This section will mainly focus on the approaches concerning the microrobot-cell interaction as it is a major domain in in-vitro applications, although these approaches can be extended to deal with other biological entities as will be shown in the application section. We categorize the interaction into three main approaches, pushing-based approach, embedded microgripper, and fluidic-based approach. Each of the three approaches offer some advantages over the other, which will be discussed as we proceed further in this section.

\section{A. Pushing-based Approach}

In this approach, the microrobot manipulates the target cell by applying a pushing force. In fact, this approach has the advantage of a relatively simplified fabrication process and actuation since the same force used for actuating the microrobot is used for pushing the cell. Nonetheless, the stable capturing of the target cell becomes more challenging due to the absence of a force field that keeps the cell attached to the microrobot.

Magnetic manipulation is a widely used actuation technique in the pushing-based approach because of its high-generated force and controllability. For example, Steager et al. [90] proposed a U-shaped magnetic microrobot for the automated manipulation of drug-carrying microbeads to deliver drugs to specified locations on neurons. Thanks to the simple shape of the microrobot with no gripping mechanism, the size could be highly miniaturized to fit in small working spaces with the advantage of generating less fluidic disturbance, which is a commonly faced problem when dealing with cells in-vitro. On the other hand, 3D actuation of microrobots could be implemented with lower complexity in fabrication compared to the other approaches. For instance, magnetic helical microrobots where fabricated with a cage-like microholder structure at one end to perform cargo transport in 3D [91]. The fabrication was conducted by a simple fabrication method using 3D direct laser writing (DLW) and actuated in a conventional manner using a system of Helmholtz coils. To achieve a more stable positioning of cells, two or more pushing forces using multiple microrobots have been proposed [92], [93], [94] (Fig. 5a(I)). For example, using two nickel microrobots actuated by permanent magnets, the on-chip positioning of oocytes was demonstrated 
TABLE I

Pros and cons of actuation techniques

\begin{tabular}{|lll|}
\hline Actuation & Pros & Cons \\
\hline Magnetic & $\begin{array}{l}\text { High dexterity; High actuation accuracy; High generated } \\
\text { force; 2D and 3D actuation }\end{array}$ & $\begin{array}{l}\text { Addressing multiple robots requires advanced techniques; } \\
\text { Low biocompatibility of most magnetic materials }\end{array}$ \\
\hline Acoustic & $\begin{array}{l}\text { High-speed propulsion in case of bubble-based designs; Bio- } \\
\text { compatible; 2D and 3D actuation }\end{array}$ & $\begin{array}{l}\text { Mostly used for directional propulsion with complementary } \\
\text { steering actuation; Addressing multiple robots requires ad- } \\
\text { vanced techniques }\end{array}$ \\
\hline Chemical & $\begin{array}{l}\text { On-board actuation; High-speed propulsion; Possible to in- } \\
\text { tegrate functionalized elements utilizing selective chemical } \\
\text { reactions }\end{array}$ & $\begin{array}{l}\text { Toxicity of hydrogen peroxide; Complementary actuation is } \\
\text { needed for steering }\end{array}$ \\
\hline Optical & $\begin{array}{l}\text { Submicrometer precision; Addressing multiple agents is fairly } \\
\text { simple; Energy conversion capability to generate thermal } \\
\text { gradients }\end{array}$ & $\begin{array}{l}\text { Low generated force; Limited working area; Possible effect on } \\
\text { biological samples when generating thermal gradients }\end{array}$ \\
\hline Biohybrid & $\begin{array}{l}\text { Biocompatible depending on the type of microorganism; On- } \\
\text { board actuation; On-board sensors using microorganism taxis }\end{array}$ & $\begin{array}{l}\text { Some microorganisms can inflict cytotoxicity; Complemen- } \\
\text { tary actuation or microorganism taxis are needed for steering }\end{array}$ \\
\hline
\end{tabular}

thanks to the millinewton-order pushing force generated by the microrobots [92]. In addition, the positioning and rotation of filamentous cells using two microrobots actuated with optical tweezers was shown [94].

Additionally, optically actuated microrobots have been developed for pushing-based single-cell positioning. Multi-traps generated by optical tweezers (OT) have been used for the 3D control of microrobots with a very high nanometric accuracy and a pushing force of $39 \mathrm{pN}$ in a master-slave setup [95]. To realize higher pushing forces with optical microrobots, microrobots driven by optoelectonic tweezers (OET) with a pushing force reaching up to $350 \mathrm{pN}$ have been proposed [96]. On the other hand, disk-shaped hydrogel microrobotic agents actuated by laser-induced microbubbles were also used for the positioning of yeast cells [93] (Fig. 5.a(II)).

Moreover, swarm microrobots are highly suitable for pushing-based approaches. They offer the advantage of utilizing collective functionality of a number of agents to access hard to reach areas and perform tasks that would be challenging to achieve by one agent. A swarm of microrobots can assemble into arbitrary shapes to navigate confined routs such as microchannels, or scatter to avoid obstacles [97], [98], [99]. For example, swarms of S. marcescens bacteria attached to $5 \sim 20 \mu \mathrm{m}$ microbeads have been controlled for payload delivery by exploiting their chemotaxis using microfluidic generated gradients of chemoattractants [100].

In summary, the pushing-based approach provides a suitable approach for cell positioning when the simplicity in fabrication and actuation are required. However, this approach relatively sacrifices the stability of cell capturing, which increases the burden on the human operator or complexifies the control algorithm.

\section{B. Embedded Microgripper}

In this approach, a gripping technique is implemented in the microrobot. It is important to note that we use the term microgripper as a universal term to indicate a structure that allows the microrobot to apply a gripping force to interact with its environment. One of the difficult challenges that arises when designing a microgripperbased microrobot is the decoupling between the motion of the microrobot and the actuation of the gripping mechanism. Therefore, a number of decoupling approaches have been implemented in the literature that can be summarized into three main categories: decoupling by actuation axis, decoupling by fabrication materials, and decoupling by using different actuation techniques, as reviewed in this section.

1) Decoupling by actuation axis: In this decoupling approach, one actuation technique is used, where the forces to move the microrobot and to actuate the gripping mechanism are axis-specific. In other words, the forces are decoupled and can be used for specific actuation of either the microrobot or the microgripper. Specifically, this approach has been mainly used in magnetically actuated microrobots, where the magnetic field generated by a permanent magnet or an electromagnet is dominant in a specific actuation plane. For example, Ichikawa et al. [101] have developed a magnetically actuated microrobot with a suction mechanism for cell positioning. The gripping mechanism was actuated on the vertical axis by incorporating an elastic Polydimethylsiloxane (PDMS) membrane covering a chamber that is connected to a micropipette (Fig. 5b). By placing a permanent magnet above the membrane and controlling its position on the vertical axis using an electromagnet, they succeeded in realizing high-power suction and pumping and the consequent on-chip positioning of an oocyte. In addition, the motion of the microrobot was realized on the horizontal plane by following the displacement of permanent magnets placed under the $\mathrm{Ni}$ plates of the microrobot. On the other hand, a similar approach is also implemented where localized actuation axes are used. In this case, both the motion of the microrobot and the actuation of the gripping mechanism are realized on the horizontal plane by following the displacement of permanent magnets placed underneath, where all the force fields are generated on the same global axis [102], [103].

The main drawback of this approach is the limitation 
in the DOF of the microrobot, mostly 3DOF (in-plane translation and rotation), because the remaining DOFs are used for the actuation of the microgripper. A solution has been proposed to achieve $4 \mathrm{DOF}$ by exploiting the balance between magnetic force and buoyancy to control the vertical position of the microrobot [103], although achieving 5DOF or higher is very challenging and have not been reported using this decoupling approach. Still, 3-4DOF actuation can be sufficient in many cases since a large number of in-vitro applications are conducted in a $2 \mathrm{D}$ environment. In addition, the interference between the force fields in different axes could be problematic and should be carefully considered. Overall, the decoupling by actuation axis still presents a DOF limited but a relatively simple approach in terms of fabrication complexity, the microrobot motion, and the gripping mechanism actuation.

2) Decoupling by fabrication materials: Another way to decouple the motion of the microrobot and the gripping mechanism is to incorporate two or more kinds of materials that would act differently when exposed to a force field or other materials. In fact, this approach offers a more robust decoupling since it relies on the reaction of materials, which are consistent under the same operating conditions. Moreover, it allows for a higher DOF actuation compared to the previous approach. However, it is crucial to choose the appropriate materials in the design phase for good performance. For instance, Diller et al. [107] have proposed a magnetic microrobot equipped with a two arms microgripper that is also magnetically actuated. The decoupling was performed by using a permanently magnetized material $(\mathrm{NdFeB})$ for one arm, and a switchable magnetic material (ferrite) for the other arm. The magnetization direction of the ferrite arm can be switched by applying a large magnetic field pulse that would result in the closing and opening of the gripper, whereas the microrobot itself was moved using low strength magnetic fields. Moreover, magnetically programmable materials have been combined with highly flexable elastomers to fabricate fingered mobile microrobots actuated only by magnetic forces and torques. Zhang et al. [108], [109] developed a four-limb star-shaped microrobot incorporating magnetic sheets with different magnetization directions. The gripping mechanism was controlled by the strength of the magnetic field, where as the motion of the microrobot was controlled by magnetic gradients, hence realizing a decoupled actuation.

Moving away from full magnetic actuation, Villa et al. [74] used superparamagnetic polymer Janus particles coated with two different materials to decouple the propelling of the particles and the capturing of the target cells, while the particles were accumulated in a chain form and steered using magnetic fields (Fig. 5c). As explained in section 2.3 , the propulsion was accomplished using a catalyst inside $\mathrm{H}_{2} \mathrm{O}_{2}$ solution. On the other hand, to allow the Janus particles to capture cells, covalent bindings were utilized through coating the particles with a porous polymeric shell that contains tosyl groups. The bindings would generate enough force to keep the cell attached to the microrobot while moving, where it is estimated that a force in the $\mathrm{pN}$ order is suitable to transport a cell of the size of several micrometers [63]. The decoupling by fabrication materials provides an attractive and a robust approach for microrobots equipped with microgrippers. The main downside of this approach would be the relatively complex fabrication process, where different materials should be integrated into one functional microrobot.

3) Decoupling by using different actuation techniques: The basic concept of this approach is to use different actuation techniques for the microrobot motion and the gripping mechanism. Recently, this novel approach is emerging due to the rapid development of new additive fabrication methods such as two-photon polymerization and selective laser sintering to name a few, which allows the fabrication of 3D microstructures. Compared to the previous two approaches, this approach offers the most robust decoupling due to the use of completely separate physical phenomena for actuation.

Magnetic actuation of a helical microrobot has been combined with optical activation of plasmonic nanostructures for a state-of-the-art cell trapping and transportation [104] (Fig. 5d(I)). The Ag plasmonic particles generate strongly localized electric fields when illuminated that results in trapping nearby targets, and rotating magnetic fields generates a propulsive motion of the helical microrobot. Trapping and releasing targets with different sizes by modifying the illumination intensity was also demonstrated. Nonetheless, the relatively low trapping force in the order of $\mathrm{fN}$ generated by the plasmonic particles limits the size of the target cell to several micrometers. On the other hand, thermally activated microgrippers have been integrated in magnetically driven microrobots [110], [105], [106]. Magdanz et al. [105] proposed a hybrid approach of biohybrid propulsion and thermal activation of reversible folding of a microtube to capture and transport a single sperm cell (Fig. 5d(II)). The microtube structure made of a thermoresponsive polymer (PNIPAM), titanium, and iron layers exhibits a folding behavior thanks to the change in hydrophobicity of PNIPAM in different temperatures. The propulsion of the microtube was achieved by the trapped sperm itself and steered using magnetic fields to the target oocyte. Although the transportable target cell can be of several tens of micrometer, the application is limited to sperm cells. Moreover, the non-local nature of the used thermal heating can reduce the biocompatibility.

Optical actuation offers a much localized actuation technique that can increase selectivity and biocompatibility. Villangca et al. [113] proposed a 3D printed fourlegged optical microrobot with a pump-like functionality actuated using optical traps in a fluidic environment. The pump-like structure is implemented using a metalcoated cavity, which generates convection flows by optothermal heating to suck or push nearby microbeads. 

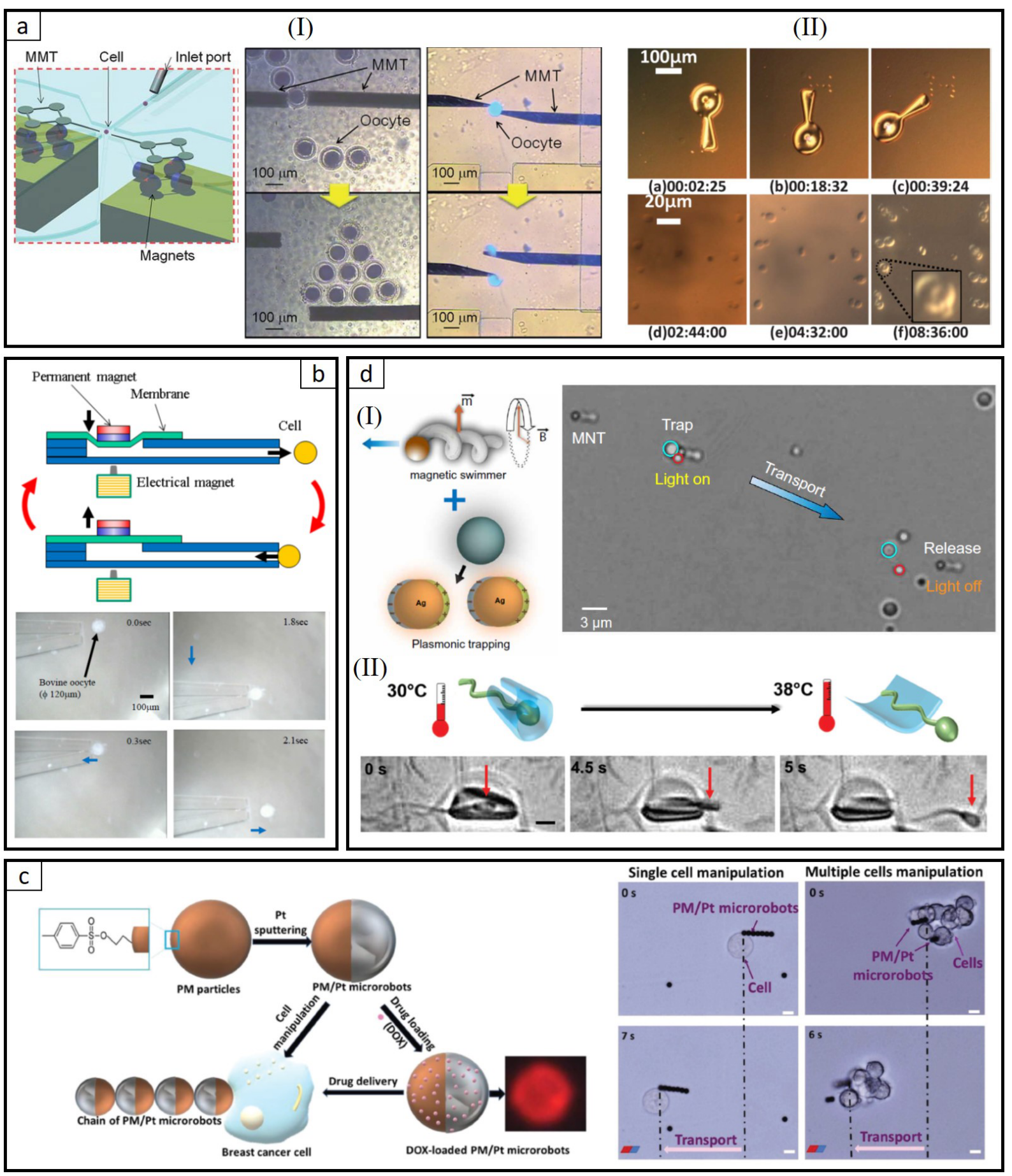

Fig. 5. (a) Pushing-based approach. (I) Cell positioning and cutting using magnetically actuated microrobots (reprinted with permission from[92]). (II) Yeast cells positioning using bubble-based optically actuated microrobot (reprinted with permission from [93]). (b-d) Embedded microgripper. (b) The decoupling by actuation axis. The microrobot equipped with a micropipette is actuated in the $\mathrm{x}-\mathrm{y}$ plane and the microgripper is actuated in the $\mathrm{z}$-axis using an electromagnet that pulls a permanent magnet placed on a membrane to generate a suction or a pushing force (reprinted with permission from [102]). (c) The decoupling by fabrication materials. The concept of coating the Janus particles with two different materials to decouple the propelling of the particles and the capturing of the target cells. The particles were propelled chemically and steered magnetically with a cell capturing technique using covalent bindings to manipulate breast cancer cells (reprinted with permission from [74]). (d) The decoupling by using different actuation techniques. (I) The concept of integrating a magnetic helical microswimmer with plasmonic Ag particles that under optical illumination generate an electrical field to trap targets and the demonstration of transporting microbeads (reprinted with permission from [104]). (II) The concept of sperm capturing using thermally foldable microtubes and the demonstration of a sperm-cell capture, transport, and release (reprinted with permission from [105], [106]). 


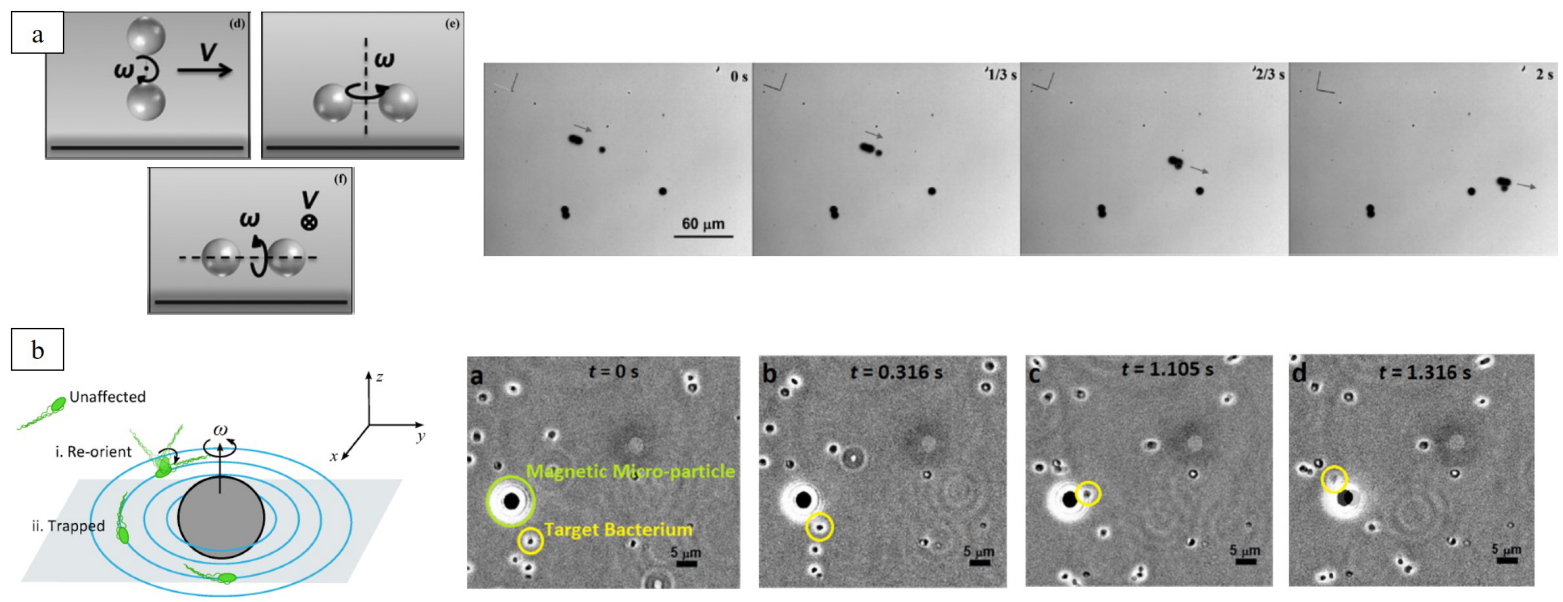

Fig. 6. Fluidic-based approach. (a) Dumbbell-like magnetic microrobot consisting of two microbeads and a nanowire. The microrobot is capable of tumbling, wobbling, and rolling motions to trap and transport microobjects using fluidic flows (reprinted with permission from [111]). (b) Magnetically actuated sphere-shape microrobot that can trap and transfer motile cells using fluidic flow (reprinted with permission from [112]).

Moreover, a hybrid approach using optothermal heating and acoustic vibrations to generate and actuate bubbles that can selectively capture and transport microbeads depending on their size was developed [114]. To select specific targets the microbubble was excited at its resonance frequency resulting in attracting large microbeads $(\phi=100 \mu \mathrm{m})$ and repelling small ones $(\phi=10 \mu \mathrm{m})$. Although optical-based approaches usually suffer from low force generation, the utilization of the optothermal effect greatly increases the generated force. Moreover, the highly localized nature of laser heating allows for a more biocompatible approach.

While this approach offers the most robust decoupling between the microrobot and the microgripper actuation, the models are developed taking into account one actuation method at a time, where coupling could occur in terms of the effect of one actuation method on the other (ex. the effect of temperature on the magnetic properties of the material). Therefore, new models that take into account the coupling between the actuation methods should be developed, which might also open the door for new designs and control methodologies. Overall, the decoupling by using different actuation techniques approach proves to be a promising and a highly robust approach and could be the solution for future mobile microrobots for in-vitro applications, although more developments are required in modeling and fabrication, where printing using different types of resins [115] could provide a boost in the functionality of the microrobots.

\section{Fluidic-based Approach}

Because cells and biological samples are usually studied in a fluidic environment, trapping and handling by inducing fluidic forces is a very common approach in the literature. In contrast to the previous two approaches, the fluidic-based approach applies a fluidic flow induced by the microrobot to trap a nearby target cell with no direct physical contact reducing cell contamination.
Moreover, the difficulty in releasing the manipulated cell is drastically reduced, where van der Waals forces and surface forces between the microrobot and the cell are not present.

Magnetically rotating microrobots are an attractive approach due to the relative simplicity of generating a rotational motion using rotating magnetic fields. The rotational movement of the microrobot around its axis induces a vortex-like fluidic flow in its vicinity that can trap a nearby target cell or a group of cells [111], [117], [118], [119] (Fig. 6a). For example, Paris et al. [116] proposed a bi-helical multistage microrobot for onchip applications. The unique design of the microrobot allows it to trap target objects in different specified locations using fluid flows and also to translate with a spintop movement or a rolling movement to allow the microrobot to access more confined areas with high dexterity in 3D. Furthermore, the merit of having no physical contact with the target cells makes the fluidicbased approach more suitable to trap and manipulate motile cells and bacteria, which can be difficult using the previous two approaches. Ye et al. [112] proposed a dynamic trapping technique of motile microorganisms by making use of a rotational flow field generated by a sphere-shape rotating magnetic microrobot (Fig. $6 \mathrm{~b})$. Their approach achieved 2D selective trapping and transportation of freely swimming bacteria successfully. The shear stress was approximately $0.4 \mathrm{~Pa}$ causing no apparent damage to cells. However, the effect of shear stress varies greatly according to the characteristics of the cells such as type, age, and the presence of a cell wall that determines its susceptibility for damage or rupture [120]. Therefore, in this approach the effect of the shear stress on vulnerable cells should be carefully considered. Moreover, the fluidic forces are induced around the whole body of the microrobot, which increases the difficulty of selective trapping of individual cells.

Table II summarizes all the discussed interaction tech- 


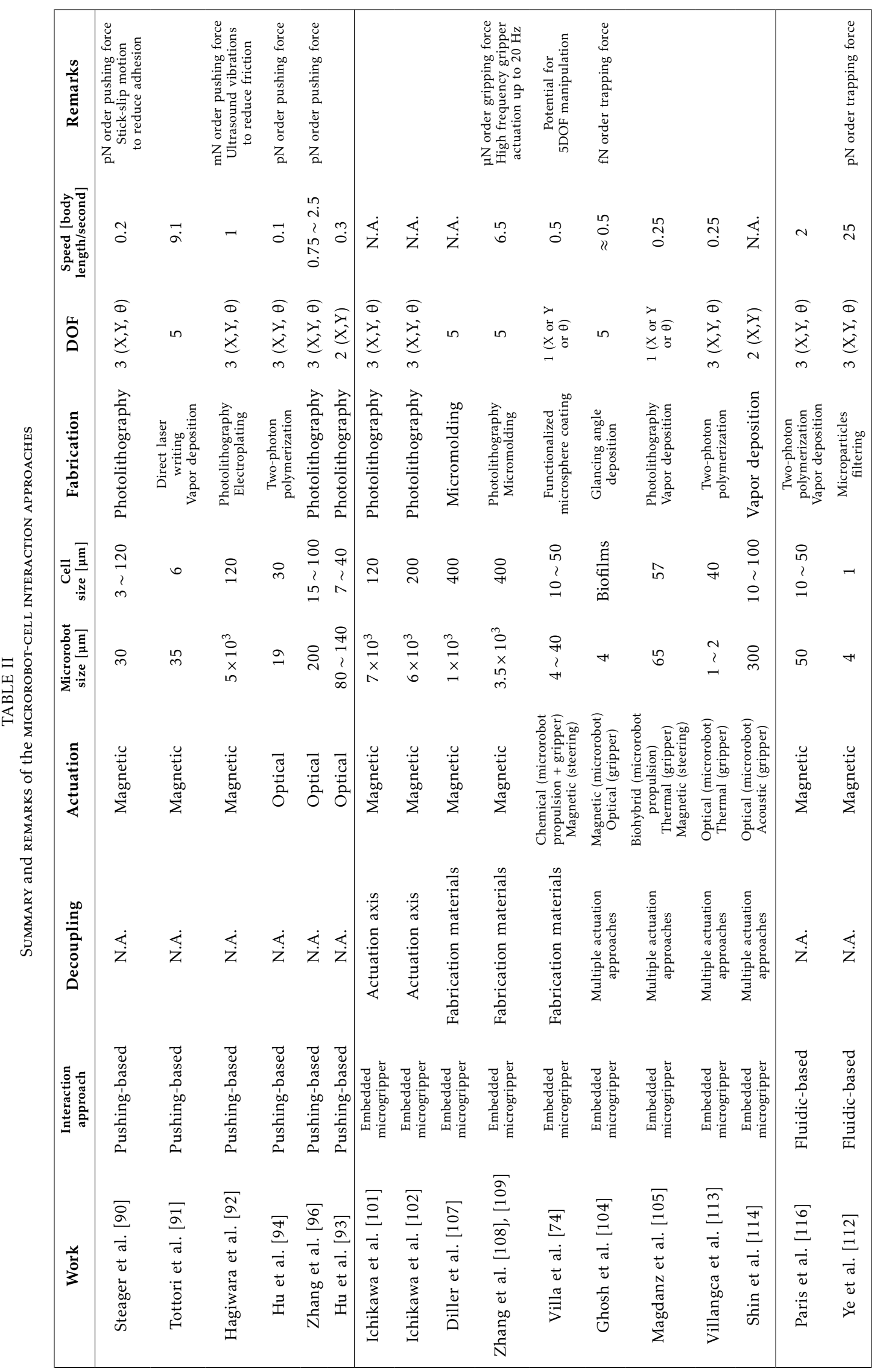


niques between the microrobot and the target cell including technical information for performance comparison. Although a variety of approaches have been implemented to develop mobile microrobots, some problems still exist that need to be solved in the future. These limitations will be further discussed in Section V.

\section{Biomedical Applications}

The use of mobile microrobots is showing a large potential for in-vitro biomedical applications. In this section, we will introduce some of the highly impactful applications in order to demonstrate the advantages offered by using mobile microrobots.

\section{A. Cell Characterization and Sensing}

The characterization of cells to elucidate their behaviours and properties especially on the single-cell level is an essential aspect in biomedical fields such as new drug discovery. Therefore, microrobots have been developed to define cells mechanical characteristics as it requires a physical contact with the cell. In fact, the high repeatability and throughout offered by mobile microrobots is highly required in such applications to reduce errors by acquiring a large amount of experimental data. Most of the currently developed sensing techniques utilize the measurement of the deformation in the integrated sensing structure. For example, Jing et al [121] developed a mobile microrobot equipped by a microforce sensor with a sensing range of $0-20 \mu \mathrm{N}$ and a resolution of approximately $1.5 \mu \mathrm{N}$, while having a positioning accuracy of $1.5 \mu \mathrm{m}$ for cell positioning and sensing. By calibrating the stiffness of the microforce sensor, real-time, closed loop, and force controlled manipulation of microparticles was demonstrated. This kind of devices is especially useful for the safe positioning of cells by indicating the applied force to avoid damage or rupture. Moreover, the use of mobile microrobots assisted the understanding and quantification of the stimulus-response of cells that could not be achieved before. Kawahara et al. [122] proposed a microrobot that can apply millinewton-order force and is equipped with a force-sensing structure to estimate the applied mechanical stimulation force on microorganisms (Fig. 7a). By quantitative evaluation of the stimulus-response of Pleurosira laevis (P. laevis), a relationship between the applied force and the single-cell response was confirmed and the stimulus required to trigger the agglomeration behaviour in these cells was determined for the first time ever.

\section{B. Cell Sorting and Positioning}

The sorting and positioning of cells is becoming one of the most fundamental and important processes in biomedical applications such as drug discovery, regenerative medicine, and innovative medicine in which the patient cells act as the drug itself. Among the different positioning tasks, sorting of a specific kind of cells from a mixture is essential for analysis and observation. To date, microcytometers and miniaturized cell sorters have been widely developed with sorting speeds reaching up to 100,000 cells/s [127]. However, these devices are designed to operate on a specific kind of cells in terms of size and speed, which limits their application. Therefore, the versatility and high selectivity of microrobots in terms of target size and speed have been used for selective single-cell transfer [128]. In this work, the microrobot showed potential for performing sorting tasks that prioritize selectivity over throughput, which are difficult to conduct using conventional cell sorters. Still, the sorting throughput of mobile microrobots is orders of magnitude lower than conventional cell sorters and more breakthroughs are required in this domain.

On the other hand, cell positioning that includes transporting and rotating the cell is highly required for cell characterization and multidirectional observations for $3 \mathrm{D}$ reconstruction tasks. This kind of tasks were conventionally conducted manually using micropipettes. Therefore, these tasks suffered from low throughput and difficulty in performing dexterous manipulations such as rotations of cells due to the tethered nature of the micropipettes. Consequently, the ability of microrobots to access confined spaces with high dexterity enables practitioners to approach cells from different directions and angles in both 2D and 3D environments. In this regard, non-contact transportation of single cells using vortexes generated by rotating or oscillating microrobots were shown [129], where successful experiments were also demonstrated for comparatively large cells such as oocytes [123] (Fig. 7b). Additionally, the in-plane and out-of-plane rotations were reported using dual arm acoustically levitated magnetic microrobots on a mammalian oocyte [130].

\section{Cell Surgery and Targeted Delivery}

Cell surgery are operations on single-cells including cutting, injecting, and extraction that are used in tasks such as enucleation and targeted drug delivery. The enucleation of an oocyte is a fundamental step in the process of cloning. Conventional methods suffer from low repeatability and throughput, a higher percentage of contamination, and require highly skilled operators working with micromanipulators. The process of cloning could become less laborious and erroneous by using microrobotic technology. For example, Ichikawa et al. [124] have demonstrated an on-chip approach using two mobile magnetic microrobots acting as a microholder and a microknife for the enucleation of an oocyte with zona pellucida (Fig. $7 \mathrm{c}(\mathrm{I}))$. Thanks to the high positioning accuracy $(5 \mu \mathrm{m})$ and the relatively large opening range of the gripper (approx. $0 \sim 160 \mu \mathrm{m}$ ), the proposed approach proved to be substantially simpler than conventional enucleation using micromanipulators and showed a large potential for cloning applications with a viability of $100 \%$ of the enucleated oocytes. On the other 


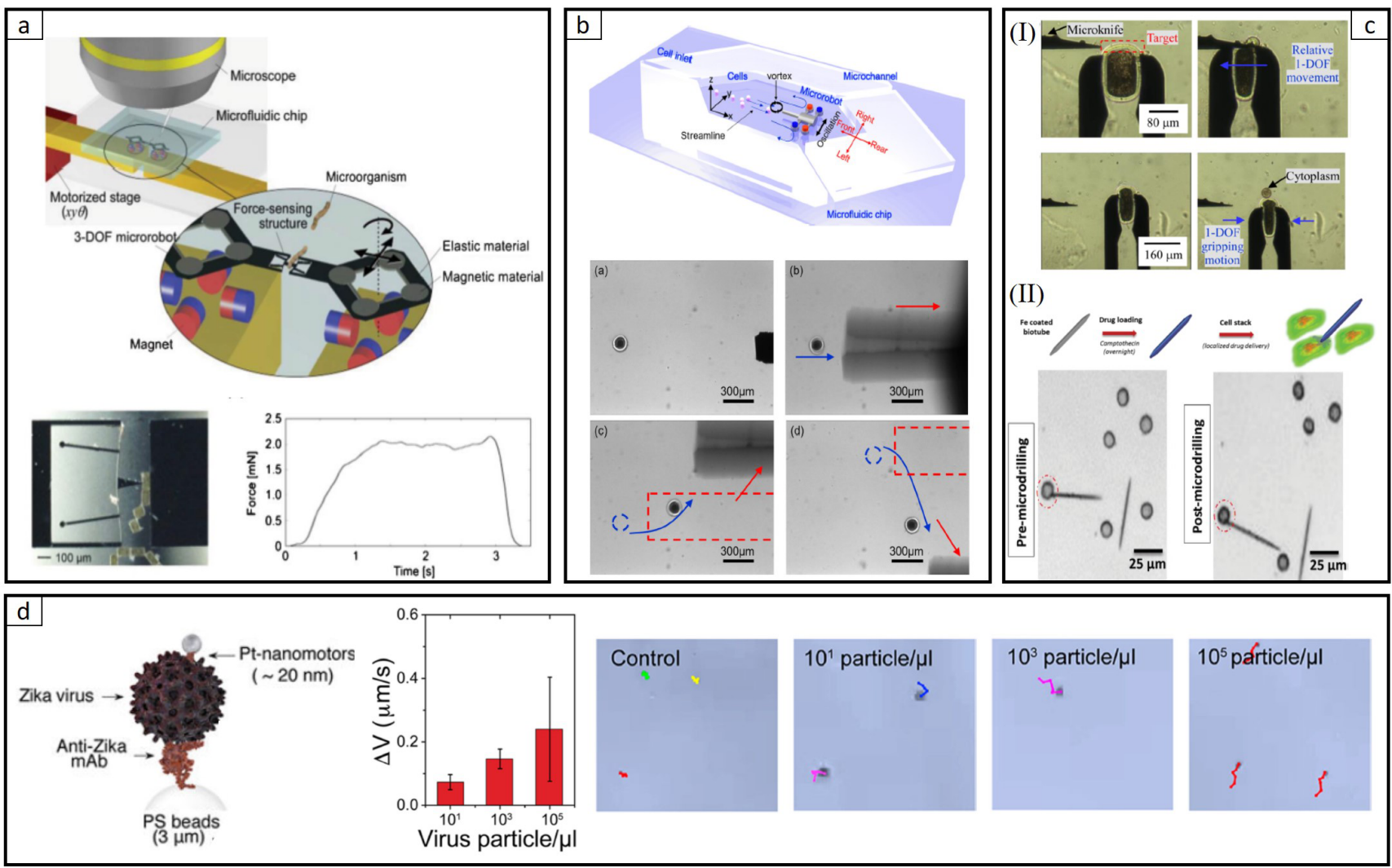

Fig. 7. Cell applications. (a) Cell sensing. A force sensing structure is attached to a mobile microrobot to apply mechanical stimulation to a microorganism with the desired force (reprinted with permission from [122]). (b) Cell handling. Trapping and positioning of an oocyte using local vortexes induced by oscillating microrobot equipped with a microtool (reprinted with permission from [123]).(c) Cell surgery. (I) The enucleation of an oocyte using two microrobots equipped with a microholder and a microknife (reprinted with permission from [124]). (II) Drilling of single cell using a drug-loaded magnetically rotating microdagger for targeted drug delivery (reprinted with permission from [125]). (d) Pathogen sensing. Zika virus sensing using nanomotors attached to a microbead through the Zika virus to generate a 3D immunocomplex that moves with a velocity proportional to the concentration of the virus (reprinted with permission from [126]).

hand, the ability to be functionalized and navigate small spaces allow the microrobot to penetrate through tissues to reach a specified location for targeted drug delivery. For instance, a novel dual-action microdagger that is composed of a $60 \mu \mathrm{m}$ long porous microneedle of a plant coated with a magnetic layer and loaded with a drug was developed for targeted drug delivery to a single HeLa cancer cell (Fig. 7c(II)) [125]. The microdagger exhibits a drilling motion when exposed to a rotating magnetic field that allows the microrobot to move with a rotational speed of approximately $146 \mathrm{rad} / \mathrm{s}$ and drill inside the cell to release the drug locally. The selective release of the drug was enabled thanks to the acidic nature $(\mathrm{pH}$ 5.8-7.6) of tumors.

On the other hand, in some biomedical applications, localized delivery of specific chemical or biological elements to individual cells on a subcellular resolution can be an essential step. These applications include proteins, genes, and drug delivery that can be utilized for cancer treatment, and to facilitate our understanding of cell-cell communication [131], [132], [133]. Qui et al. [132] conducted gene delivery to human embryonic kidney cells using a functionalized magnetic microswimmer. The microswimmers loaded with plasmid DNA and cationic lipids were actuated using rotating magnetic fields. This allowed the swimmer to get in contact with the target cell and deliver DNA, in which the lipids helped to facilitate the DNA fusion with the cell. Moreover, protein delivery with subcellular resolution was realized using cytokine-carrying Au nanowires [133]. The $6 \mu \mathrm{m}$ long nanowires were actuated by electric tweezers to approach and stimulate HeLa cells, where the stimulus response and the cell signaling was confirmed.

\section{Pathogens Sensing and Targeting}

The study of pathogens and toxins is an important aspect of pathology that allow us to analyze the possible causes, transmission, and possible cures of a certain disease. Specifically, the early detection, evaluation, and elimination of such pathogens with high accuracy is essential to realize a rapid and effective treatment [134]. Currently used techniques, such as biological assays for detection and broad-spectrum antibiotic for elimination, tend to be costly, time consuming, and non-localized causing latency in diagnosis and reduced effectiveness in treatment [135]. The mobility and ability to functionalize microrobots together with its low cost fabrication 
enable the deployment of fast and versatile mobile sensors, while allowing targeted drug delivery. Therefore, mobile microrobots have been recently developed for pathogen sensing [136], [126] and targeted delivery to kill pathogens [137], [138], [139]. Draz et al., [126] introduced a novel system to detect the concentration of Zika virus inside a microfluidic chip based on tracking the nanomotor-induced motion of microbeads (Fig. 7). The platinum nanomototrs and the microbeads $(\phi=3$ $\mu \mathrm{m})$ are treated with anti-Zika virus monoclona antibodies to facilitate a connection between the nanomotors and the microbeads in the presence of the Zika virus. By virtue of the antibodies, the higher the number of virus particles in the medium, the more nanomotors will connect to each microbead. Thus, the concentration of the virus can be measured by tracking the average velocity of the microbeads. On the other hand, Stanton et al. [137] proposed a biohybrid magnetic microrobot consisting of magnetotactic bacteria integrated inside a drug-loaded silica microtube for targeted killing of E. coli biofilms colonies (Fig. $7 \mathrm{~d}(\mathrm{II})$ ). The $10 \sim 20 \mu \mathrm{m}$-long biohybrid microrobots were propelled and magnetically steered towards the target biofilm colony by virtue of the magnetically guidable bacteria with a velocity reaching up to $8 \mu \mathrm{m} / \mathrm{s}$.

\section{Outlook and Future Direction}

The drastic advancements and breakthroughs in mobile microrobots have demonstrated significant merits in in-vitro biomedical applications. Nonetheless, the use of mobile microrobots for biological experiments has been almost limited to demonstrational purposes, whereas the vast majority of works in the biomedical field are conducted using the conventional methods. Still, there exists a large room for improvements and breakthroughs in miniaturization, functionality, and autonomy.

\section{A. Miniaturization}

The majority of mobile microrobots for in-vitro applications are designed to deal with biological entities ranging in size from few up to several hundreds of micrometers. In fact, sensing the activity of a cell or investigating a specific structure inside of it is of high importance to understand cell-cell interaction and their stimulus response. For instance, extracellular vesicles (e.g., exosomes), which are membranous structures with a size ranging from tens of nanometers up to few micrometers, play an important role during the communication from one cell to another. Therefore, the sensing and investigation of a single extracellular vesicle is of high interest for medical applications such as cancer monitoring and treatment [140], [141], although a better understanding of their functions and behaviors is still needed. Devices such as AFM and optical tweezers are capable of handling targets in this size range [142], although they fall short in dexterity and maneuverability compared to mobile microrobots due to their tethered nature. Nanomotors and nanorobots offer a good candidate for tasks at the nanoscale such as nanosensing and handling submicrometer objects [126], [143], [144]. However, dexterous submicrometric tasks such as electrical sensing, cutting, and injection are still not achievable using such nanorobots. In particular, the current state of the art in sensing using mobile microrobots is limited to mechanical sensing such as cell stiffness that does not require nanoscale functional elements in the microrobot [121], [122]. Therefore, combining the dexterity of microrobots with the submicron sensing capabilities of nanorobots could provide a versatile tool capable of intracellular and extracellular sensing and handling. Therefore, further miniaturization of microrobots while maintaining their dexterity and functionality is required to handle such extremely small targets. Recently, this is more enabled by the emergence of additive microfabrication techniques such as photon polymerization and selective laser sintering, which could permit the integration of functional parts within the nanometric scale.

\section{B. Functionality and Performance}

Handling biological entities safely with high precision and high speed is one of the remarkable advantages offered by mobile microrobots. In terms of safety, biological entities should be handled with care due to their delicacy and research have been conducted to integrate mechanical sensing structures in microrobots for cell handling [121]. Still, the relatively rigid structures of conventional microrobots is a suboptimal approach, hence the current trend is shifting towards utilizing functional soft materials as a promising alternative for safe handling [143]. In terms of precision, despite achieving high positioning accuracy of the microrobot, the position of the handled cell still suffers from inaccuracies mainly in the release process due to the adhesion between the microrobot and the handled cell. In general, to achieve the release of a handled cell, the microrobot is actuated away from the cell to overcome the adhesion forces with fluidic forces, or a non-contact fluidic-based approach is deployed to handle the cell, although the precision of the cell's final position is still affected [90]. Therefore, it is necessary to develop releasing techniques to achieve precise release of cells. In terms of speed, the main bottleneck is the target cell itself, since its position is substantially sensitive to fluidic flows. The high speed actuation of the microrobot inside the fluidic environment generates large fluidic disturbances that would affect the position of all the cells in its vicinity, including the target cell. For instance, in [93] the speed of the microrobot had to be reduced more than ten times to minimize the fluidic disturbances on cells. Therefore, promising workarounds could include the fabrication of microrobots with hydrodynamic shapes, and the modeling and estimation of the fluidic flows generated by the movement of the microrobot [37] in an attempt to minimize or compensate the disturbances. 
On the other hand, biocompatibility and biodegradability of microrobots are an essential challenge towards their deployment in the biomedical industry. A number of biocompatible microrobots have been developed using techniques including biocompatible materials, coating, and biohybrid designs [3], [145], [146], [147]. For instance, Alcantara et al. [147] evaluated the cytotoxicity of iron microrobots by culturing them with human colorectal cancer cells for four days with no signs of cytotoxicity. In contrast, less attention is paid to the biodegradability of microrobots for in-vitro applications compared to their in-vivo counterparts because they are used outside the human body. However, biodegradability has been demonstrated using metal-organic frameworks microrobots that are guided magnetically, where the microrobots degraded fully after 14 days inside the cell culture [148]. Still, biocompatibility and biodegradability need further development and investigation on a larger scale for microrobots to be incorporated as standardized tools in biomedical applications.

\section{Autonomy and Intelligence}

The future direction of mobile microrobots is aiming towards having fully autonomous and intelligent microrobots capable of environment sensing and decision making to perform tasks that are demanding using the current technology, which relies mostly on teleportation-based actuation. This new generation of intelligent microrobots would allow the access of further confined, obscured, and hard to reach areas within the working space such as microchannels. Moreover, the ability to sense and interact with the environment would enable the microrobot to handle delicate, and force-sensitive biological cells in an efficient and safe manner and would allow for autonomous collaboration between robotic agents and swarms. The current state of the art is exploring intelligence in microrobots in two domains, software intelligence (i.e. external sensing, decision making, and control), and hardware intelligence, also known as physical intelligence (i.e. internal sensing, decision making, and control). In software intelligence, because many mobile microrobots are actuated using feedback from external vision sensors, extended autonomy would start from detailed sensing and analysis of the surrounding environment and objects [8], [90], [109], [149]. Consequently, an intelligent control algorithm that incorporates a rigorous and adaptive model of the microrobot dynamics could enable autonomous path planning, navigation, obstacle avoidance, and fine interaction with the target cells [150], [151]. For instance, Pawashe et al. [150] developed a mobile microrobot that is able to autonomously manipulate and assemble microobjects in fluids. In this case, the effect of the fluidic drag caused by the motion of the microrobot had to be modeled and incorporated in the feedback control algorithm enabling successful path planning and the prediction of the resultant motion of the microobjects to achieve the assembly task. Furthermore, automation at the swarm level have been demonstrated on a snakelike microrobotic swarm [152]. By modeling the forces acting on the swarm, programmed pattern generation utilizing a genetic algorithm was achieved. The swarm was capable of navigating unknown environments using automated path generation and simultaneous localization and mapping.

On the contrary, hardware intelligence is based on encoding the intelligence within the microrobot by the integration of materials sensitive to the environmental changes that initiate responses to different stimulus cues [106], [153]. This domain is especially promising for chemically and biohybrid actuated microrobots by exploiting selective, or thermally-sensitive chemical reactions, or the different taxis and stimulus-responses of microorganisms [100], [154]. For instance, Kim et al. propose a novel approach that combines software and hardware intelligence for obstacle avoidance of a bacteria-powered microrobot by exploiting the response of bacteria to electrical field [155]. Because the presence of obstacles distorts the applied electric field and disrupts the control, an objective function for the distortion in the electric field was utilized to detect and navigate obstacles. However, both software and hardware intelligence are still immature and more work is required to pave the way towards fully autonomous mobile microrobots.

Overall, although further research is required to overcome the current limitations, mobile microrobots are highly promising for in-vitro applications and offer a strong candidate to facilitate revolutionary breakthroughs in the biomedical fields.

\section{REFERENCES}

[1] C. Chautems, B. Zeydan, S. Charreyron, G. Chatzipirpiridis, S. Pane, and B. J. Nelson, "Magnetically powered microrobots: a medical revolution underway?," European Journal of CardioThoracic Surgery, vol. 51, no. 3, pp. 405-407, 2017.

[2] D. Jang, J. Jeong, H. Song, and S. K. Chung, "Targeted drug delivery technology using untethered microrobots: A review," Journal of Micromechanics and Microengineering, vol. 29, no. 5, p. $053002,2019$.

[3] J. Li, X. Li, T. Luo, R. Wang, C. Liu, S. Chen, D. Li, J. Yue, S.-h. Cheng, and D. Sun, "Development of a magnetic microrobot for carrying and delivering targeted cells," Science Robotics, vol. 3, no. $19,2018$.

[4] B. Ahmad, T. Kawahara, T. Yasuda, and F. Arai, "Microrobotic platform for mechanical stimulation of swimming microorganism on a chip," in 2014 IEEE/RSJ International Conference on Intelligent Robots and Systems, pp. 4680-4685, 2014.

[5] C. Hu and L. Li, "Preconditioning influences mesenchymal stem cell properties in vitro and in vivo," Journal of cellular and molecular medicine, vol. 22, no. 3, pp. 1428-1442, 2018.

[6] A. V. Singh, M. H. D. Ansari, M. Mahajan, S. Srivastava, S. Kashyap, P. Dwivedi, V. Pandit, and U. Katha, "Sperm cell driven microrobots-emerging opportunities and challenges for biologically inspired robotic design," Micromachines, vol. 11, no. 4 , p. 448,2020

[7] M. Sitti and D. S. Wiersma, "Pros and cons: Magnetic versus optical microrobots," Advanced Materials, vol. 32, no. 20, p. $1906766,2020$. 
[8] M. Dkhil, M. Kharboutly, A. Bolopion, S. Régnier, and M. Gauthier, "Closed-loop control of a magnetic particle at the airliquid interface," IEEE Transactions on Automation Science and Engineering, vol. 14, no. 3, pp. 1387-1399, 2015.

[9] S. Chowdhury, W. Jing, and D. J. Cappelleri, "Towards independent control of multiple magnetic mobile microrobots," Micromachines, vol. 7, no. 1, p. 3, 2016

[10] Z. Wu, L. Li, Y. Yang, P. Hu, Y. Li, S.-Y. Yang, L. V. Wang, and W. Gao, "A microrobotic system guided by photoacoustic computed tomography for targeted navigation in intestines in vivo," Science robotics, vol. 4, no. 32, 2019.

[11] D. Li, D. Dong, W. Lam, L. Xing, T. Wei, and D. Sun, "Automated in vivo navigation of magnetic-driven microrobots using oct imaging feedback," IEEE Transactions on Biomedical Engineering, vol. 67 , no. 8 , pp. $2349-2358,2020$.

[12] A. Pena-Francesch, J. Giltinan, and M. Sitti, "Multifunctional and biodegradable self-propelled protein motors," Nature communications, vol. 10, no. 1, pp. 1-10, 2019.

[13] M. Sitti, H. Ceylan, W. Hu, J. Giltinan, M. Turan, S. Yim, and E. Diller, "Biomedical applications of untethered mobile milli/microrobots," Proceedings of the IEEE, vol. 103, no. 2, pp. 205-224, 2015.

[14] C. Yin, F. Wei, Z. Zhan, J. Zheng, L. Yao, W. Yang, and M. Li, "Untethered microgripper-the dexterous hand at microscale," Biomedical Microdevices, vol. 21, no. 4, p. 82, 2019.

[15] B. J. Nelson, I. K. Kaliakatsos, and J. J. Abbott, "Microrobots for minimally invasive medicine," Annual review of biomedical engineering, vol. 12 , pp. 55-85, 2010.

[16] S. Martel, "Bacterial microsystems and microrobots," Biomedical microdevices, vol. 14, no. 6, pp. 1033-1045, 2012.

[17] Y. Alapan, O. Yasa, B. Yigit, I. C. Yasa, P. Erkoc, and M. Sitti, "Microrobotics and microorganisms: Biohybrid autonomous cellular robots," Annual Review of Control, Robotics, and Autonomous Systems, vol. 2, pp. 205-230, 2019.

[18] P. Erkoc, I. C. Yasa, H. Ceylan, O. Yasa, Y. Alapan, and M. Sitti, "Mobile microrobots for active therapeutic delivery," Advanced Therapeutics, vol. 2, no. 1, p. 1800064, 2019

[19] H. Ceylan, J. Giltinan, K. Kozielski, and M. Sitti, "Mobile microrobots for bioengineering applications," Lab on a Chip, vol. 17, no. 10, pp. 1705-1724, 2017.

[20] S. Palagi and P. Fischer, "Bioinspired microrobots," Nature Reviews Materials, vol. 3, no. 6, p. 113, 2018.

[21] K. E. Peyer, L. Zhang, and B. J. Nelson, "Bio-inspired magnetic swimming microrobots for biomedical applications," Nanoscale, vol. 5, no. 4, pp. 1259-1272, 2013.

[22] K. Svennersten, M. Berggren, A. Richter-Dahlfors, and E. W. Jager, "Mechanical stimulation of epithelial cells using polypyrrole microactuators," Lab on a Chip, vol. 11, no. 19, pp. 32873293, 2011

[23] L. Dedelaite, R. D. Rodriguez, E. Andriukonis, M. Hietschold D. R. Zahn, and A. Ramanavicius, "Surfaces functionalized by graphene oxide nanosheets for single cell investigations," Sensors and Actuators B: Chemical, vol. 255, pp. 1735-1743, 2018.

[24] B. Ahmad, H. Maeda, and T. Kawahara, "Dynamic response of swimming paramecium induced by local stimulation using a threadlike-microtool," IEEE Robotics and Automation Letters, vol. 5, no. 2, pp. 2570-2577, 2020.

[25] Q. Zeb, C. Wang, S. Shafiq, and L. Liu, "An overview of singlecell isolation techniques," in Single-Cell Omics, pp. 101-135, Elsevier, 2019.

[26] M. H. Spitzer and G. P. Nolan, "Mass cytometry: single cells, many features," Cell, vol. 165, no. 4, pp. 780-791, 2016

[27] M. P. Hughes, "Fifty years of dielectrophoretic cell separation technology," Biomicrofluidics, vol. 10, no. 3, p. 032801, 2016.

[28] I. C. Yasa, A. F. Tabak, O. Yasa, H. Ceylan, and M. Sitti, "3dprinted microrobotic transporters with recapitulated stem cell niche for programmable and active cell delivery," Advanced Functional Materials, vol. 29, no. 17, p. 1808992, 2019.

[29] E. Avci, M. Grammatikopoulou, and G.-Z. Yang, "Laser-printing and 3d optical-control of untethered microrobots," Advanced Optical Materials, vol. 5, no. 19, p. 1700031, 2017.

[30] J. F. Schenck, "Safety of strong, static magnetic fields," Journal of magnetic resonance imaging, vol. 12, no. 1, pp. 2-19, 2000.

[31] M. P. Kummer, J. J. Abbott, B. E. Kratochvil, R. Borer, A. Sengul, and B. J. Nelson, "Octomag: An electromagnetic system for 5dof wireless micromanipulation," IEEE Transactions on Robotics, vol. 26 , no. 6 , pp. 1006-1017, 2010.
[32] E. Diller, J. Giltinan, G. Z. Lum, Z. Ye, and M. Sitti, "Six-degreeof-freedom magnetic actuation for wireless microrobotics," The International Journal of Robotics Research, vol. 35, no. 1-3, pp. 114-128, 2016.

[33] A. Alasli, L. Çetin, N. Akçura, A. Kahveci, F. C. Can, and Ö. Tamer, "Electromagnet design for untethered actuation system mounted on robotic manipulator," Sensors and Actuators A: Physical, vol. 285, pp. 550-565, 2019.

[34] A. Bolopion, S. Bouchebout, and S. Régnier, "Fast, repeatable and precise magnetic actuation in ambient environments at the micrometer scale," Journal of Micro-Bio Robotics, vol. 13, no. 1-4, pp. 55-66, 2017.

[35] G. Hwang, I. A. Ivan, J. Agnus, H. Salmon, S. Alvo, N. Chaillet, S. Régnier, and A.-M. Haghiri-Gosnet, "Mobile microrobotic manipulator in microfluidics," Sensors and Actuators A: Physical, vol. 215, pp. 56-64, 2014.

[36] H. Kim, J. Ali, U. K. Cheang, J. Jeong, J. S. Kim, and M. J. Kim, "Micro manipulation using magnetic microrobots," Journal of Bionic Engineering, vol. 13, no. 4, pp. 515-524, 2016.

[37] S. Floyd, C. Pawashe, and M. Sitti, "Two-dimensional contact and noncontact micromanipulation in liquid using an untethered mobile magnetic microrobot," IEEE Transactions on Robotics, vol. 25, no. 6, pp. 1332-1342, 2009.

[38] S. Kim, S. Hashi, and K. Ishiyama, "Magnetic actuation based snake-like mechanism and locomotion driven by rotating magnetic field," IEEE transactions on magnetics, vol. 47, no. 10, pp. 3244-3247, 2011.

[39] E. Al Khatib, A. Bhattacharjee, P. Razzaghi, L. W. Rogowski, M. J. Kim, and Y. Hurmuzlu, "Magnetically actuated simple millirobots for complex navigation and modular assembly," IEEE Robotics and Automation Letters, vol. 5, no. 2, pp. 2958-2965, 2020.

[40] M. A. Zeeshan, R. Grisch, E. Pellicer, K. M. Sivaraman, K. E. Peyer, J. Sort, B. Özkale, M. S. Sakar, B. J. Nelson, and S. Pané, "Hybrid helical magnetic microrobots obtained by $3 \mathrm{~d}$ templateassisted electrodeposition," Small, vol. 10, no. 7, pp. 1284-1288, 2014.

[41] A. Servant, F. Qiu, M. Mazza, K. Kostarelos, and B. J. Nelson, "Controlled in vivo swimming of a swarm of bacteria-like microrobotic flagella," Advanced Materials, vol. 27, no. 19, pp. 29812988, 2015.

[42] P. Katsamba and E. Lauga, "Micro-tug-of-war: A selective control mechanism for magnetic swimmers," Physical Review Applied, vol. 5, no. 6, p. 064019, 2016.

[43] J. Giltinan, P. Katsamba, W. Wang, E. Lauga, and M. Sitti, "Selectively controlled magnetic microrobots with opposing helices," Applied Physics Letters, vol. 116, no. 13, p. 134101, 2020.

[44] J. Tang, L. W. Rogowski, X. Zhang, and M. J. Kim, "Flagellar nanorobot with kinetic behavior investigation and 3d motion," Nanoscale, 2020.

[45] L. W. Rogowski, M. Oxner, J. Tang, and M. J. Kim, "Heterogeneously flagellated microswimmer behavior in viscous fluids," Biomicrofluidics, vol. 14, no. 2, p. 024112, 2020.

[46] U. K. Cheang, H. Kim, D. Milutinović, J. Choi, and M. J. Kim, "Feedback control of an achiral robotic microswimmer," Journal of Bionic Engineering, vol. 14, no. 2, pp. 245-259, 2017.

[47] S. Kim, S. Lee, J. Lee, B. J. Nelson, L. Zhang, and H. Choi, "Fabrication and manipulation of ciliary microrobots with nonreciprocal magnetic actuation," Scientific reports, vol. 6, p. 30713, 2016.

[48] I. S. Khalil, H. C. Dijkslag, L. Abelmann, and S. Misra, "Magnetosperm: A microrobot that navigates using weak magnetic fields," Applied Physics Letters, vol. 104, no. 22, p. 223701, 2014.

[49] A. M. Maier, C. Weig, P. Oswald, E. Frey, P. Fischer, and T. Liedl, "Magnetic propulsion of microswimmers with dna-based flagellar bundles," Nano letters, vol. 16, no. 2, pp. 906-910, 2016.

[50] U. K. Cheang and M. J. Kim, "Self-assembly of robotic microand nanoswimmers using magnetic nanoparticles," Journal of Nanoparticle Research, vol. 17, no. 3, pp. 1-11, 2015.

51] M. Hagiwara, T. Kawahara, Y. Yamanishi, and F. Arai, "Driving method of microtool by horizontally arranged permanent magnets for single cell manipulation," Applied Physics Letters, vol. 97, no. 1, p. 013701, 2010.

[52] P. Ryan and E. Diller, "Magnetic actuation for full dexterity microrobotic control using rotating permanent magnets," IEEE Transactions on Robotics, vol. 33, no. 6, pp. 1398-1409, 2017. 
[53] H. Tung, M. Maffioli, D. R. Frutiger, K. M. Sivaraman, S. Pané, and B. J. Nelson, "Polymer-based wireless resonant magnetic microrobots," IEEE Transactions on Robotics, vol. 30, no. 1, pp. 26-32, 2014.

[54] J. Ali, U. K. Cheang, Y. Liu, H. Kim, L. Rogowski, S. Sheckman, P. Patel, W. Sun, and M. J. Kim, "Fabrication and magnetic control of alginate-based rolling microrobots," AIP Advances, vol. 6 , no. 12 , p. $125205,2016$.

[55] L. Ren, N. Nama, J. M. McNeill, F. Soto, Z. Yan, W. Liu, W. Wang, J. Wang, and T. E. Mallouk, "3d steerable, acoustically powered microswimmers for single-particle manipulation," Science advances, vol. 5, no. 10, p. eaax3084, 2019.

[56] M. Kaynak, A. Ozcelik, A. Nourhani, P. E. Lammert, V. H. Crespi, and T. J. Huang, "Acoustic actuation of bioinspired microswimmers," Lab on a Chip, vol. 17, no. 3, pp. 395-400, 2017.

[57] N. Bertin, T. A. Spelman, O. Stephan, L. Gredy, M. Bouriau, E. Lauga, and P. Marmottant, "Propulsion of bubble-based acoustic microswimmers," Physical Review Applied, vol. 4, no. 6, p. 064012, 2015

[58] A. Aghakhani, O. Yasa, P. Wrede, and M. Sitti, "Acoustically powered surface-slipping mobile microrobots," Proceedings of the National Academy of Sciences, vol. 117, no. 7, pp. 3469-3477, 2020.

[59] J. Jeong, D. Jang, D. Kim, D. Lee, and S. K. Chung, "Acoustic bubble-based drug manipulation: Carrying, releasing and penetrating for targeted drug delivery using an electromagnetically actuated microrobot," Sensors and Actuators A: Physical, p. 111973, 2020.

[60] D. Ahmed, T. Baasch, B. Jang, S. Pane, J. Dual, and B. J. Nelson, "Artificial swimmers propelled by acoustically activated flagella," Nano letters, vol. 16, no. 8, pp. 4968-4974, 2016.

[61] C. Fei, Y. Li, B. Zhu, C. T. Chiu, Z. Chen, D. Li, Y. Yang, K. Kirk Shung, and Q. Zhou, "Contactless microparticle control via ultrahigh frequency needle type single beam acoustic tweezers," Applied Physics Letters, vol. 109, no. 17, p. 173509, 2016.

[62] A. A. Solovev, W. Xi, D. H. Gracias, S. M. Harazim, C. Deneke, S. Sanchez, and O. G. Schmidt, "Self-propelled nanotools," Acs Nano, vol. 6, no. 2, pp. 1751-1756, 2012.

[63] J. Wang, "Self-propelled affinity biosensors: Moving the receptor around the sample," Biosensors and Bioelectronics, vol. 76, pp. 234-242, 2016.

[64] M. Luo, Y. Feng, T. Wang, and J. Guan, "Micro-/nanorobots at work in active drug delivery," Advanced Functional Materials, vol. 28 , no. 25 , p. $1706100,2018$.

[65] M. Manjare, B. Yang, and Y.-P. Zhao, "Bubble-propelled microjets: Model and experiment," The Journal of Physical Chemistry C, vol. 117, no. 9, pp. 4657-4665, 2013.

[66] N. Hu, M. Sun, X. Lin, C. Gao, B. Zhang, C. Zheng, H. Xie, and Q. He, "Self-propelled rolled-up polyelectrolyte multilayer microrockets," Advanced Functional Materials, vol. 28, no. 25, p. 1705684, 2018.

[67] H. Ceylan, I. C. Yasa, and M. Sitti, "3d chemical patterning of micromaterials for encoded functionality," Advanced Materials, vol. 29, no. 9, p. 1605072, 2017.

[68] B. Jurado-Sanchez, M. Pacheco, R. Maria-Hormigos, and A. Escarpa, "Perspectives on janus micromotors: Materials and applications," Applied Materials Today, vol. 9, pp. 407-418, 2017.

[69] X. Ma, S. Jang, M. N. Popescu, W. E. Uspal, A. MiguelLopez, K. Hahn, D.-P. Kim, and S. Sanchez, "Reversed janus micro/nanomotors with internal chemical engine," ACS nano, vol. 10, no. 9, pp. 8751-8759, 2016.

[70] D. Yamamoto and A. Shioi, "Self-propelled nano/micromotors with a chemical reaction: underlying physics and strategies of motion control," KONA Powder and Particle Journal, p. 2015005, 2015.

[71] I. S. Khalil, V. Magdanz, S. Sanchez, O. G. Schmidt, and S. Misra, "Wireless magnetic-based closed-loop control of self-propelled microjets," PloS one, vol. 9, no. 2, p. e83053, 2014.

[72] L. W. Rogowski, X. Zhang, L. Huang, A. Bhattacharjee, J. S. Lee, A. T. Becker, and M. J. Kim, "Feedback control and 3d motion of heterogeneous janus particles," in IEEE International Conference on Robotics and Automation (ICRA), pp. 1352-1357, 2019.

[73] T. Xu, F. Soto, W. Gao, V. Garcia-Gradilla, J. Li, X. Zhang, and J. Wang, "Ultrasound-modulated bubble propulsion of chemi- cally powered microengines," Journal of the American Chemical Society, vol. 136, no. 24, pp. 8552-8555, 2014.

[74] K. Villa, L. Krejčová, F. Novotnỳ, Z. Heger, Z. Sofer, and M. Pumera, "Cooperative multifunctional self-propelled paramagnetic microrobots with chemical handles for cell manipulation and drug delivery," Advanced Functional Materials, vol. 28, no. 43, p. 1804343, 2018.

[75] M. Medina-Sánchez, H. Xu, and O. G. Schmidt, "Micro-and nano-motors: the new generation of drug carriers," Therapeutic delivery, vol. 9, no. 4, pp. 303-316, 2018.

[76] M. A. Rahman, J. Cheng, Z. Wang, and A. T. Ohta, "Cooperative micromanipulation using the independent actuation of fifty microrobots in parallel," Scientific reports, vol. 7, no. 1, pp. 1-11, 2017.

[77] H. Xu, M. Medina-Sánchez, V. Magdanz, L. Schwarz, F. Hebenstreit, and O. G. Schmidt, "Sperm-hybrid micromotor for targeted drug delivery," ACS nano, vol. 12, no. 1, pp. 327-337, 2018.

[78] T. Wu, T. A. Nieminen, S. Mohanty, J. Miotke, R. L. Meyer, H. Rubinsztein-Dunlop, and M. W. Berns, "A photon-driven micromotor can direct nerve fibre growth," nature photonics, vol. 6, no. 1, pp. 62-67, 2012.

[79] R. T. Mallea, A. Bolopion, J.-C. Beugnot, P. Lambert, and M. Gauthier, "Closed-loop particle motion control using laser-induced thermocapillary convective flows at the fluid/gas interface at micrometric scale," IEEE/ASME transactions on mechatronics, vol. 23 , no. 4 , pp. $1543-1554,2018$.

[80] Q. Fan, W. Hu, and A. T. Ohta, "Laser-induced microbubble poration of localized single cells," Lab on a Chip, vol. 14, no. 9, pp. 1572-1578, 2014.

[81] S. Palagi, A. G. Mark, S. Y. Reigh, K. Melde, T. Qiu, H. Zeng, C. Parmeggiani, D. Martella, A. Sanchez-Castillo, N. Kapernaum, et al., "Structured light enables biomimetic swimming and versatile locomotion of photoresponsive soft microrobots," Nature materials, vol. 15, no. 6, pp. 647-653, 2016.

[82] O. Ilic, I. Kaminer, Y. Lahini, H. Buljan, and M. Soljacic, "Exploiting optical asymmetry for controlled guiding of particles with light," Acs Photonics, vol. 3, no. 2, pp. 197-202, 2016.

[83] B. Behkam and M. Sitti, "Bacterial flagella-based propulsion and on/off motion control of microscale objects," Applied Physics Letters, vol. 90, no. 2, p. 023902, 2007.

[84] D. Li, H. Choi, S. Cho, S. Jeong, Z. Jin, C. Lee, S. Y. Ko, J.O. Park, and S. Park, "A hybrid actuated microrobot using an electromagnetic field and flagellated bacteria for tumortargeting therapy," Biotechnology and bioengineering, vol. 112, no. 8, pp. 1623-1631, 2015.

[85] D. B. Weibel, P. Garstecki, D. Ryan, W. R. DiLuzio, M. Mayer, J. E. Seto, and G. M. Whitesides, "Microoxen: Microorganisms to move microscale loads," Proceedings of the National Academy of Sciences, vol. 102, no. 34, pp. 11963-11967, 2005.

[86] K. Huh, D. Oh, H. J. Yoo, B. Song, D.-i. D. Cho, J.-M. Seo, and S. J. Kim, "Bacteria-based microrobot for chemotaxis delivery," in 2015 15th International Conference on Control, Automation and Systems (ICCAS), pp. 1848-1852, IEEE, 2015.

[87] H. J. Yoo, S. Lee, and D.-i. D. Cho, "Motility control of bacteriaactuated biodegradable polymeric microstructures by selective adhesion methods," Micromachines, vol. 5, no. 4, pp. 1287-1295, 2014.

[88] R. W. Carlsen, M. R. Edwards, J. Zhuang, C. Pacoret, and M. Sitti, "Magnetic steering control of multi-cellular bio-hybrid microswimmers," Lab on a Chip, vol. 14, no. 19, pp. 3850-3859, 2014.

[89] M. M. Stanton, B.-W. Park, A. Miguel-López, X. Ma, M. Sitti, and S. Sánchez, "Biohybrid microtube swimmers driven by single captured bacteria," Small, vol. 13, no. 19, p. 1603679, 2017.

[90] E. B. Steager, M. Selman Sakar, C. Magee, M. Kennedy, A. Cowley, and V. Kumar, "Automated biomanipulation of single cells using magnetic microrobots," The International Journal of Robotics Research, vol. 32, no. 3, pp. 346-359, 2013.

[91] S. Tottori, L. Zhang, F. Qiu, K. K. Krawczyk, A. Franco-Obregón, and B. J. Nelson, "Magnetic helical micromachines: fabrication, controlled swimming, and cargo transport," Advanced materials, vol. 24, no. 6, pp. 811-816, 2012.

[92] M. Hagiwara, T. Kawahara, Y. Yamanishi, T. Masuda, L. Feng, and F. Arai, "On-chip magnetically actuated robot with ultrasonic vibration for single cell manipulations," Lab on a Chip, vol. 11, no. 12, pp. 2049-2054, 2011. 
[93] W. Hu, K. S. Ishii, Q. Fan, and A. T. Ohta, "Hydrogel microrobots actuated by optically generated vapour bubbles," Lab on a Chip, vol. 12, no. 19, pp. 3821-3826, 2012.

[94] S. Hu, R. Hu, X. Dong, T. Wei, S. Chen, and D. Sun, "Translational and rotational manipulation of filamentous cells using optically driven microrobots," Optics express, vol. 27, no. 12, pp. 16475-16482, 2019.

[95] E. Gerena, F. Legendre, A. Molawade, Y. Vitry, S. Régnier, and S. Haliyo, "Tele-robotic platform for dexterous optical singlecell manipulation," Micromachines, vol. 10, no. 10, p. 677, 2019.

[96] S. Zhang, E. Y. Scott, J. Singh, Y. Chen, Y. Zhang, M. Elsayed, M. D. Chamberlain, N. Shakiba, K. Adams, S. Yu, et al., "The optoelectronic microrobot: A versatile toolbox for micromanipulation," Proceedings of the National Academy of Sciences, vol. 116, no. 30, pp. 14823-14828, 2019.

[97] X. Zhang, L. W. Rogowski, and M. J. Kim, "3d micromanipulation of particle swarm using a hexapole magnetic tweezer," in IEEE/RSJ International Conference on Intelligent Robots and Systems (IROS), 2019.

[98] H. Xie, M. Sun, X. Fan, Z. Lin, W. Chen, L. Wang, L. Dong, and Q. He, "Reconfigurable magnetic microrobot swarm: Multimode transformation, locomotion, and manipulation," Sci. Robot, vol. 4, no. 28, 2019.

[99] B. Yigit, Y. Alapan, and M. Sitti, "Programmable collective behavior in dynamically self-assembled mobile microrobotic swarms," Advanced Science, vol. 6, no. 6, p. 1801837, 2019.

[100] D. Kim, A. Liu, E. Diller, and M. Sitti, "Chemotactic steering of bacteria propelled microbeads," Biomedical microdevices, vol. 14, no. 6, pp. 1009-1017, 2012.

[101] A. Ichikawa and F. Arai, "Magnetically driven micro-robot with suction mechanism for on-chip automatic," in 2012 IEEE International Conference on Automation Science and Engineering (CASE), pp. 273-278, IEEE, 2012.

[102] A. Ichikawa, S. Sakuma, F. Arai, and S. Akagi, "Untethered micro-robot with gripping mechanism for on-chip cell surgery utilizing outer magnetic force," in 2014 IEEE International Conference on Robotics and Automation (ICRA), pp. 3795-3800, IEEE, 2014.

[103] L. Feng, X. Wu, Y. Jiang, D. Zhang, and F. Arai, "Manipulating microrobots using balanced magnetic and buoyancy forces," Micromachines, vol. 9, no. 2, p. 50, 2018.

[104] S. Ghosh and A. Ghosh, "Mobile nanotweezers for active colloidal manipulation," Science Robotics, vol. 3, no. 14, 2018.

[105] V. Magdanz, M. Guix, F. Hebenstreit, and O. G. Schmidt, "Dynamic polymeric microtubes for the remote-controlled capture, guidance, and release of sperm cells," Advanced materials, vol. 28, no. 21, pp. 4084-4089, 2016.

[106] V. Magdanz, M. Medina-Sánchez, L. Schwarz, H. Xu, J. Elgeti, and O. G. Schmidt, "Spermatozoa as functional components of robotic microswimmers," Advanced Materials, vol. 29, no. 24, p. 1606301, 2017.

[107] E. Diller and M. Sitti, "Three-dimensional programmable assembly by untethered magnetic robotic micro-grippers," Advanced Functional Materials, vol. 24, no. 28, pp. 4397-4404, 2014.

[108] J. Zhang and E. Diller, "Tetherless mobile micrograsping using a magnetic elastic composite material," Smart Materials and Structures, vol. 25, no. 11, p. 11LT03, 2016.

[109] J. Zhang, O. Onaizah, K. Middleton, L. You, and E. Diller, "Reliable grasping of three-dimensional untethered mobile magnetic microgripper for autonomous pick-and-place," IEEE Robotics and Automation Letters, vol. 2, no. 2, pp. 835-840, 2017.

[110] Q. Jin, Y. Yang, J. A. Jackson, C. Yoon, and D. H. Gracias, "Untethered single cell grippers for active biopsy," Nano Letters, vol. 20, no. 7, pp. 5383-5390, 2020. PMID: 32463679.

[111] Q. Zhou, T. Petit, H. Choi, B. J. Nelson, and L. Zhang, "Dumbbell fluidic tweezers for dynamical trapping and selective transport of microobjects," Advanced Functional Materials, vol. 27, no. 1, p. 1604571, 2017.

[112] Z. Ye and M. Sitti, "Dynamic trapping and two-dimensional transport of swimming microorganisms using a rotating magnetic microrobot," Lab on a Chip, vol. 14, no. 13, pp. 2177-2182, 2014.

[113] M. J. Villangca, D. Palima, A. R. Banas, and J. Glückstad, "Lightdriven micro-tool equipped with a syringe function," Light: Science \& Applications, vol. 5, no. 9, pp. e16148-e16148, 2016.
[114] J. H. Shin, J. Seo, J. Hong, and S. K. Chung, "Hybrid optothermal and acoustic manipulations of microbubbles for precise and ondemand handling of micro-objects," Sensors and Actuators B: Chemical, vol. 246, pp. 415-420, 2017.

[115] Z.-C. Ma, Y.-L. Zhang, B. Han, X.-Y. Hu, C.-H. Li, Q.-D. Chen, and H.-B. Sun, "Femtosecond laser programmed artificial musculoskeletal systems," Nature communications, vol. 11, no. 1, pp. 1-10, 2020.

[116] A. Paris, D. Decanini, and G. Hwang, "On-chip multimodal vortex trap micro-manipulator with multistage bi-helical microswimmer," Sensors and Actuators A: Physical, vol. 276, pp. 118124, 2018.

[117] L. Zhang, T. Petit, K. E. Peyer, and B. J. Nelson, "Targeted cargo delivery using a rotating nickel nanowire," Nanomedicine: Nanotechnology, Biology and Medicine, vol. 8, no. 7, pp. 1074 1080,2012

[118] H.-W. Tung, K. E. Peyer, D. F. Sargent, and B. J. Nelson, "Noncontact manipulation using a transversely magnetized rolling robot," Applied Physics Letters, vol. 103, no. 11, p. 114101, 2013.

[119] I. S. M. Khalil, A. Klingner, Y. Hamed, Y. S. Hassan, and S. Misra, "Controlled noncontact manipulation of nonmagnetic untethered microbeads orbiting two-tailed soft microrobot," IEEE Transactions on Robotics, vol. 36, no. 4, pp. 1320-1332, 2020.

[120] Y. Chisti, "Hydrodynamic damage to animal cells," Critical reviews in biotechnology, vol. 21, no. 2, pp. 67-110, 2001.

[121] W. Jing, S. Chowdhury, M. Guix, J. Wang, Z. An, B. V. Johnson, and D. J. Cappelleri, "A microforce-sensing mobile microrobot for automated micromanipulation tasks," IEEE Transactions on Automation Science and Engineering, vol. 16, no. 2, pp. 518-530, 2018.

[122] T. Kawahara, M. Sugita, M. Hagiwara, F. Arai, H. Kawano, I. Shihira-Ishikawa, and A. Miyawaki, "On-chip microrobot for investigating the response of aquatic microorganisms to mechanical stimulation," Lab on a Chip, vol. 13, no. 6, pp. 10701078, 2013.

[123] L. Feng, S. Liang, X. Zhou, J. Yang, Y. Jiang, D. Zhang, and F. Arai, "On-chip microfluid induced by oscillation of microrobot for noncontact cell transportation," Applied Physics Letters, vol. 111 , no. 20 , p. $203703,2017$.

[124] A. Ichikawa, S. Sakuma, M. Sugita, T. Shoda, T. Tamakoshi, S. Akagi, and F. Arai, "On-chip enucleation of an oocyte by untethered microrobots," Journal of Micromechanics and Microengineering, vol. 24, no. 9, p. 095004, 2014.

[125] S. K. Srivastava, M. Medina-Sánchez, B. Koch, and O. G. Schmidt, "Medibots: dual-action biogenic microdaggers for single-cell surgery and drug release," Advanced Materials, vol. 28, no. 5, pp. 832-837, 2016.

[126] M. S. Draz, N. K. Lakshminaraasimulu, S. Krishnakumar, D. Battalapalli, A. Vasan, M. K. Kanakasabapathy, A. Sreeram, S. Kallakuri, P. Thirumalaraju, Y. Li, et al., "Motion-based immunological detection of zika virus using pt-nanomotors and a cellphone," ACS nano, vol. 12, no. 6, pp. 5709-5718, 2018.

[127] J. Picot, C. L. Guerin, C. Le Van Kim, and C. M. Boulanger, "Flow cytometry: retrospective, fundamentals and recent instrumentation," Cytotechnology, vol. 64, no. 2, pp. 109-130, 2012.

[128] M. Hagiwara, T. Kawahara, T. Iijima, and F. Arai, "High-speed magnetic microrobot actuation in a microfluidic chip by a fine v-groove surface," IEEE Transactions on Robotics, vol. 29, no. 2, pp. 363-372, 2012.

[129] T. Petit, L. Zhang, K. E. Peyer, B. E. Kratochvil, and B. J. Nelson, "Selective trapping and manipulation of microscale objects using mobile microvortices," Nano letters, vol. 12, no. 1, pp. 156-160, 2012.

[130] L. Feng, P. Di, and F. Arai, "High-precision motion of magnetic microrobot with ultrasonic levitation for 3-d rotation of single oocyte," The International Journal of Robotics Research, vol. 35, no. 12 , pp. $1445-1458,2016$.

[131] B.-W. Park, J. Zhuang, O. Yasa, and M. Sitti, "Multifunctional bacteria-driven microswimmers for targeted active drug delivery," ACS nano, vol. 11, no. 9, pp. 8910-8923, 2017.

[132] F. Qiu, S. Fujita, R. Mhanna, L. Zhang, B. R. Simona, and B. J. Nelson, "Magnetic helical microswimmers functionalized with lipoplexes for targeted gene delivery," Advanced Functional Materials, vol. 25, no. 11, pp. 1666-1671, 2015.

[133] D. Fan, Z. Yin, R. Cheong, F. Q. Zhu, R. C. Cammarata, C. Chien, and A. Levchenko, "Subcellular-resolution delivery of a cytokine 
through precisely manipulated nanowires," Nature nanotechnology, vol. 5, no. 7, pp. 545-551, 2010.

[134] A. M. Foudeh, T. F. Didar, T. Veres, and M. Tabrizian, "Microfluidic designs and techniques using lab-on-a-chip devices for pathogen detection for point-of-care diagnostics," Lab on a Chip, vol. 12, no. 18, pp. 3249-3266, 2012.

[135] P. Babaie, A. Saadati, and M. Hasanzadeh, "Recent progress and challenges on the bioassay of pathogenic bacteria," Journal of Biomedical Materials Research Part B: Applied Biomaterials, 2020.

[136] Y. Zhang, L. Zhang, L. Yang, C. I. Vong, K. F. Chan, W. K. Wu, T. N. Kwong, N. W. Lo, M. Ip, S. H. Wong, et al., "Real-time tracking of fluorescent magnetic spore-based microrobots for remote detection of c. diff toxins," Science advances, vol. 5, no. 1, p. eaau9650, 2019.

[137] M. M. Stanton, B.-W. Park, D. Vilela, K. Bente, D. Faivre, M. Sitti, and S. Sanchez, "Magnetotactic bacteria powered biohybrids target e. coli biofilms," ACS nano, vol. 11, no. 10, pp. 99689978, 2017.

[138] C. Chen, L. Chen, P. Wang, L.-F. Wu, and T. Song, "Steering of magnetotactic bacterial microrobots by focusing magnetic field for targeted pathogen killing," Journal of Magnetism and Magnetic Materials, vol. 479, pp. 74-83, 2019.

[139] C. Chen, L. Chen, P. Wang, L.-F. Wu, and T. Song, "Magneticallyinduced elimination of staphylococcus aureus by magnetotactic bacteria under a swing magnetic field," Nanomedicine: Nanotechnology, Biology and Medicine, vol. 13, no. 2, pp. 363-370, 2017.

[140] F. Urabe, N. Kosaka, Y. Yoshioka, S. Egawa, and T. Ochiya, "The small vesicular culprits: the investigation of extracellular vesicles as new targets for cancer treatment," Clinical and Translational Medicine, vol. 6, no. 1, p. 45, 2017.

[141] Y. Liu, C. Xu, P. Yu, X. Chen, J. Wang, and L. Mao, "Counting and sizing of single vesicles/liposomes by electrochemical events," ChemElectroChem, vol. 5, no. 20, pp. 2954-2962, 2018.

[142] M. Li, N. Xi, Y. Wang, and L. Liu, "Progress in nanorobotics for advancing biomedicine," IEEE Transactions on Biomedical Engineering, pp. 1-1, 2020.

[143] C. Hu, S. Pané, and B. J. Nelson, "Soft micro-and nanorobotics," Annual Review of Control, Robotics, and Autonomous Systems, vol. 1, pp. 53-75, 2018.

[144] A. Halder and Y. Sun, "Biocompatible propulsion for biomedical micro/nano robotics," Biosensors and Bioelectronics, vol. 139, p. 111334, 2019.

[145] K. Villa, J. Viktorova, J. Plutnar, T. Ruml, L. Hoang, and M. Pumera, "Chemical microrobots as self-propelled microbrushes against dental biofilm," Cell Reports Physical Science, vol. 1, no. 9, p. 100181, 2020.

[146] G. Go, A. Yoo, H.-W. Song, H.-K. Min, S. Zheng, K. T. Nguyen, S. Kim, B. Kang, A. Hong, C.-S. Kim, et al., "Multifunctional biodegradable microrobot with programmable morphology for biomedical applications," ACS nano, 2020.

[147] C. C. Alcântara, S. Kim, S. Lee, B. Jang, P. Thakolkaran, J.-Y. Kim, H. Choi, B. J. Nelson, and S. Pané, "3d fabrication of fully iron magnetic microrobots," Small, vol. 15, no. 16, p. 1805006, 2019.

[148] A. Terzopoulou, X. Wang, X.-Z. Chen, M. Palacios-Corella, C. Pujante, J. Herrero-Martín, X.-H. Qin, J. Sort, A. J. deMello, B. J. Nelson, et al., "Biodegradable metal-organic framework-based microrobots (mofbots)," Advanced Healthcare Materials, vol. 9, no. 20, p. 2001031, 2020.

[149] J. Kim, H. Choi, and J. Kim, "A robust motion control with antiwindup scheme for electromagnetic actuated microrobot using time-delay estimation," IEEE/ASME Transactions on Mechatronics, vol. 24, no. 3, pp. 1096-1105, 2019.

[150] C. Pawashe, S. Floyd, E. Diller, and M. Sitti, "Two-dimensional autonomous microparticle manipulation strategies for magnetic microrobots in fluidic environments," IEEE Transactions on Robotics, vol. 28, no. 2, pp. 467-477, 2011.

[151] L. Yang, Y. Zhang, Q. Wang, K.-F. Chan, and L. Zhang, "Automated control of magnetic spore-based microrobot using fluorescence imaging for targeted delivery with cellular resolution," IEEE Transactions on Automation Science and Engineering, vol. 17, no. 1 , pp. $490-501,2019$.

[152] H. Xie, X. Fan, M. Sun, Z. Lin, Q. He, and L. Sun, "Programmable generation and motion control of a snakelike magnetic microrobot swarm," IEEE/ASME Transactions on Mechatronics, vol. 24, no. 3, pp. 902-912, 2019.
[153] Y. Tu, F. Peng, X. Sui, Y. Men, P. B. White, J. C. van Hest, and D. A. Wilson, "Self-propelled supramolecular nanomotors with temperature-responsive speed regulation," Nature chemistry, vol. 9, no. 5, pp. 480-486, 2017.

[154] P. S. S. Kim, A. Becker, Y. Ou, A. A. Julius, and M. J. Kim "Imparting magnetic dipole heterogeneity to internalized iron oxide nanoparticles for microorganism swarm control," Journal of Nanoparticle Research, vol. 17, no. 3, p. 144, 2015.

[155] H. Kim and M. J. Kim, "Electric field control of bacteria-powered microrobots using a static obstacle avoidance algorithm," IEEE Transactions on Robotics, vol. 32, no. 1, pp. 125-137, 2015.

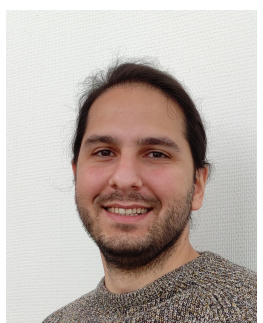

Belal Ahmad received his MSc and Ph.D. in engineering from Kyushu Institute of Technology, Kitakyushu, Japan, in 2015 and 2019, respectively. He is currently a postdoctoral researcher at the Automatic Control and MicroMechatronic Systems Department, FEMTO-ST, Besancon, France.

His main research interests are microrobotics, robotic systems integration, and high-speed tracking.

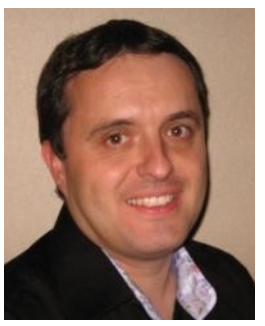

Michaël Gauthier CNRS senior scientist, works in the field of micro-nanorobotics since 2000 and has proposed, modeled and experimented new robotics tools for microhandling, micromanipulation and micro-assembly in several European and National projects. He is the president of the French-Swiss Alliance between FEMTOST and EPFL named SMYLE.

During the last years, he was the head of the micro-nanorobotics department in FEMTOST (2012-16) and vice-director of FEMTO-ST institute (2016-19). He is the author of 2 books, more than 45 papers and 60 conference talks. He is also the co-founder of the spin-off 'Percipio Robotics' providing micro-assembly platforms for industrial applications.

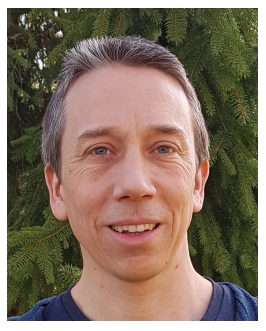

Guillaume J. Laurent received the Ph.D. degree in control systems and computer sciences from the University of Franche-Comte, Besancon, France, in 2002. He is currently an Associate Professor with the National School of Mechanics and Microtechnologies (ENSMM), Besancon.

$\mathrm{He}$ is a member of the Automatic Control and Micro-Mechatronic Systems Department, FEMTO-ST Institute. His research interests include microrobotics, computer vision, parallel, and continuum robots.

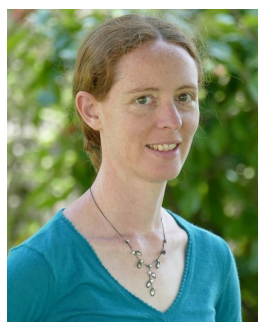

Aude Bolopion received her Ph.D. degree in robotics in 2010 from Sorbonne University (ex. Pierre et Marie Curie University), Paris, France. She was a member of the ISIR Institute. Her research interests were focused on teleoperation and haptic feedback at the nanoscale.

She got a CNRS researcher permanent position at the FEMTO-ST Institute, Besancon, France in 2011. She received the CNRS bronze medal in 2019 for her work on non contact actuation for microrobotics. 